\title{
A Review of Distributed Fiber-optic Sensing in the Oil and Gas Industry
}

\author{
Islam Ashry, Senior Member, IEEE, Yuan Mao, Senior Member, IEEE, Biwei Wang, Frode Hveding, \\ Ahmed Y. Bukhamsin, Tien Khee Ng, Senior Member, IEEE, and Boon S. Ooi
}

\author{
(Invited Paper)
}

\begin{abstract}
Fiber-optic sensors have been widely deployed in various applications, and their use has gradually increased since the 1980s. Distributed fiber-optic sensors, which enable continuous and real-time measurements along the entire length of an optical fiber cable, have undergone significant improvements in underlying industries. In the oil and gas industry, distributed fiberoptic sensors can provide significantly valuable information throughout the life cycle of a well and can monitor pipelines transporting hydrocarbons over great distances. Here, we review the deployment of fiber-optic Rayleighbased distributed acoustic sensing (DAS), Raman-based distributed temperature sensing (DTS), and Brillouin-based distributed temperature and strain sensing (DTSS) in the oil and gas industry. In particular, we describe the operation principle and basic experimental setups of the DAS, DTS, and DTSS, highlighting their applications in the upstream, midstream, and downstream sectors of the oil and gas industry. We further developed a prototype of a fiber-optic hybrid DAS-DTS system that simultaneously measures vibration and temperature along a multimode fiber (MMF). The reported hybrid sensing system was tested in an operational oil well. This work also discusses the challenges that might hinder the growth of the distributed fiber-optic sensing market in the petroleum industry, and we further point out the future directions of related research.
\end{abstract}

Index Terms-fiber-optic sensing, distributed acoustic sensing, distributed temperature sensing, distributed strain sensing, oil and gas industry.

\section{INTRODUCTION}

$\mathbf{I}$ NCREASING prosperity in developing countries coupled with rapid global population growth is expected to lead

Manuscript received X, 2021; revised X, 2021. This work was supported by Saudi Aramco (RGC/3/3138-01); King Abdullah University of Science and Technology (KAUST)-Research Translation Funding (REI/1/4578-01-01).

I. Ashry, Y. Mao, T. K. Ng and B. S. Ooi are with the King Abdullah University of Science and Technology (KAUST), Computer, Electrical, and Mathematical Sciences and Engineering, Thuwal 23955-6900, Saudi Arabia (e-mail: islam.ashry@kaust.edu.sa; yuan.mao@kaust.edu.sa; tienkhee.ng@kaust.edu.sa; boon.ooi@kaust.edu.sa).

B. Wang is with the Department of Electronic and Information Engineering, The Hong Kong Polytechnic University, Hong Kong, China (email: biwei.wang@connect.polyu.hk).

F. Hveding and A. Y. Bukhamsin are with the EXPEC Advanced Research Center, Saudi Aramco, Dhahran 34464, Saudi Arabia (e-mail: frode.hveding@aramco.com; ahmad.bukhamseen@aramco.com).

I. Ashry, Y. Mao, and B. Wang contributed equally to this work. to an increase in energy consumption worldwide over the coming decades. In spite of spreading awareness about energy conservation measures, expectations predict that global energy demand would increase to $725 \mathrm{EJ}$ in 2050 , compared to $575 \mathrm{EJ}$ required in 2018 [1]. In the coming decades, sustainable energy sources are anticipated to provide a significant portion of the energy required. However, renewable energy sources would not be sufficient to fully meet the expected needed energy, which requires the use of fossil fuels, nuclear power, etc., to supply the remaining energy [1].

Hydrocarbons (oil and gas) have become the world's most important source of energy [2]. For example, hydrocarbons provide fuel for vehicles, power industry, and heat homes. Besides, oil and gas are the basis of many products, including plastics, chemicals, paints, detergents, among others. Given the numerous hydrocarbons' benefits, it is unlikely that sufficient alternatives to hydrocarbons would exist in the coming decades [3]. As a result, oil and gas companies will have to continue producing adequate quantities of hydrocarbons in the decades ahead. This requires persistent optimization, improvement, monitoring, and control of the hydrocarbons' production and processing techniques.

The oil and gas industry can be divided into three major sectors, called upstream, midstream and downstream (Fig. 1) [4], [5]. The upstream sector includes all activities related to exploration and production of hydrocarbons. These activities comprise, for example, geological surveys, seismic acquisitions, prospecting, drilling, reservoir evaluation, well testing, well completion, and bringing oil and gas resources to the surface. In contrast, the midstream sector is responsible for transporting and storing raw oil and gas before refining and processing them. Hence, midstream includes separation plants, pipelines, pumping stations, tank trucks, railway tankers, etc., needed to transport hydrocarbon resources over long distances from wells to refineries. The downstream sector starts operating in the refineries that convert crude oil and gas into useful products, such as gasoline, diesel, kerosene, heating oils and plastics, to mention a few. In addition, the downstream sector also includes transporting processed products to retail distributors, which can again be done by pipelines, trucks, railways and ships.

The upstream, midstream and downstream sectors face different operational challenges. To optimize and control various 


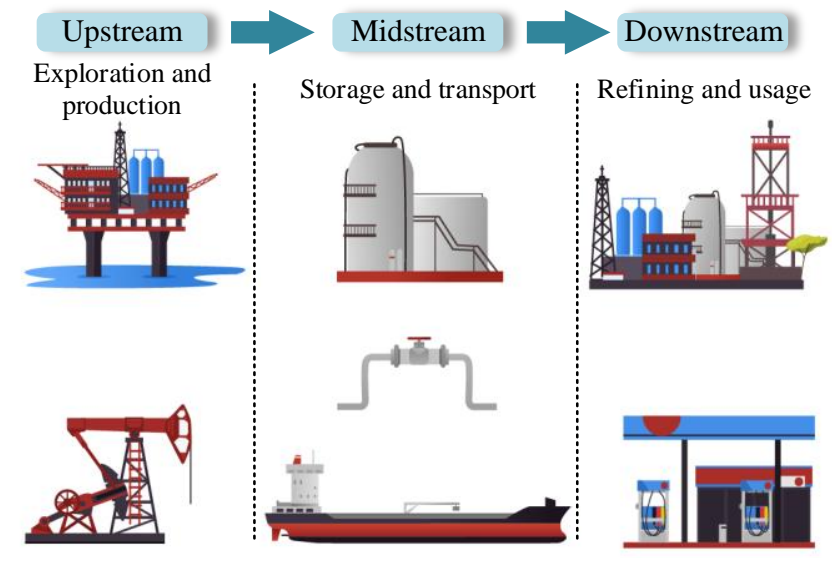

Fig. 1. Upstream, midstream, and downstream sector of the oil and gas industry.

applications within the three sectors, it is necessary to monitor various parameters, including temperature, vibration, pressure, flow, etc. To clarify, we briefly provide some representative technical examples to shed light on the challenges of downhole life and the transport of hydrocarbons through pipelines.

Early measurements made in reservoir evaluation include acquiring borehole seismic data, which can be recorded using a distributed acoustic sensing (DAS) system. This can be achieved by using a standard wireline cable containing additional optical fiber to record DAS data at the same time that the standard wireline tasks are being performed [6], [7]. The DAS borehole seismic acquisition offers data early in the life of the well to calibrate the depth of surface seismic images and thus impact decisions on well completion.

Another technical example is during the production of oil and gas, where it is important to take into account the dynamic processes that take place downhole with the objective of reducing water production [8]. In the lifetime of a producing well, an increasing amount of water may enter the wellbore, hence taking up the space for oil and gas to flow. As a result, hydrocarbon production reduces and water production increases. Having a DAS in combination with a distributed temperature sensing (DTS) downhole can identify the source of water entry [9]. DAS can be used to determine the speed of sound, that is, the speed at which a pressure wave propagates through the fluid. The speed of sound varies based on the ratio of oil, water, and gas flowing within the tubing. For example, the speed of sound is faster in water than that in oil. Thus, it is possible to extract the flowing phase and identify the water entry locations using the DAS technology. In addition, due to the pressure drop caused by fluid transition from reservoir pressure to wellbore pressure, there will be either a cooling effect if free gas is entering or a frictional heating effect if fluid is entering the wellbore. Due to changes in the thermal properties of oil, gas, and water, the continuous temperature profiles provided by the DTS system can locate the water entry points.

On the other hand, pipelines are pivotal as they provide long-distance transports of raw oil/gas to refineries and petroleum products to end-users in the midstream and downstream sector, respectively. Compared to railways, trucks, and ships, pipelines are safer, more environmentally-friendly, more cost-effective and provide continuous transportation of hydrocarbons with a larger capacity [10]. Due to the importance of pipelines, it is highly recommended to ensure pipeline integrity by monitoring leakage, deformation, and intrusion along pipelines [11]. Due to the difficulty of monitoring kilometers of pipelines with surveillance cameras and human oversight, it is preferred to use distributed sensing techniques to monitor pipelines.

The aforementioned representative examples confirm the need to sense various parameters in the oil and gas industry. However, sensors used must be designed to survive the harsh environmental conditions of that industry. This is because the downhole temperature and pressure can reach $200{ }^{\circ} \mathrm{C}$ and 2000 bar, respectively [12]. In other circumstances, such as when steam is injected into the wellbore to reduce the viscosity of heavy oil and enhance oil recovery, the downhole temperature exceeds $300{ }^{\circ} \mathrm{C}$ [12]. In addition, downhole sensors may be exposed to vibration, shock, corrosive chemicals, and fluid immersion. Besides, pipelines may transport oil/gas throughout deserts, deep seabeds, and polar regions.

Electronic-based sensors have been widely used in the oil and gas industry [13]. However, electronics is considered as one of the main reasons for failure of permanent downhole measurement and control systems [14]. The reliability of oil and gas well electronics can deteriorate gradually due mainly to elevated downhole temperature. Since the intended product lifespan in the oil and gas industry is typically five years or more [14], there are many petroleum applications where electronic sensors cannot be used at all. Additionally, electronic sensors are active devices that consume electrical energy to operate, which raises safety concerns in the oil and gas industry.

In contrast, fiber-optic sensors have been reliably deployed in many oil and gas applications [12], [15]. The success of using fiber-optic sensors in the oil and gas industry is due to the inherent characteristics of optical fibers. One main advantage of optical fibers is the ability to operate efficiently in harsh environmental conditions, which is well suited for downhole sensing applications [16]. This is achieved by extending optical fibers downhole, with the optoelectronic sensing interrogation unit located on the surface. Besides, optical fiber has immunity to electromagnetic interference, its size is miniature, and it does not need electric current at the sensing locations, which are well in line with the safety requirements of the oil and gas industry. In addition, optical fibers have the ability to monitor physical parameters over long distances, which is necessary for pipeline monitoring and downhole sensing.

Fiber-optic sensors can be classified as single-point, quasidistributed, and distributed sensors [17]. Initially, fiber-optic sensors were developed as pointwise sensors, which monitor environmental parameters at one location along the fiber. Various types of single-point fiber-optic sensors have been introduced, including grating-based sensors (fiber Bragg grating (FBG), long-period grating (LPG), etc.) and interferometric sensors (Fabry-Perot, Mach-Zehnder, etc.) [18]. Following the advancement of optical multiplexing, such as wavelength- 


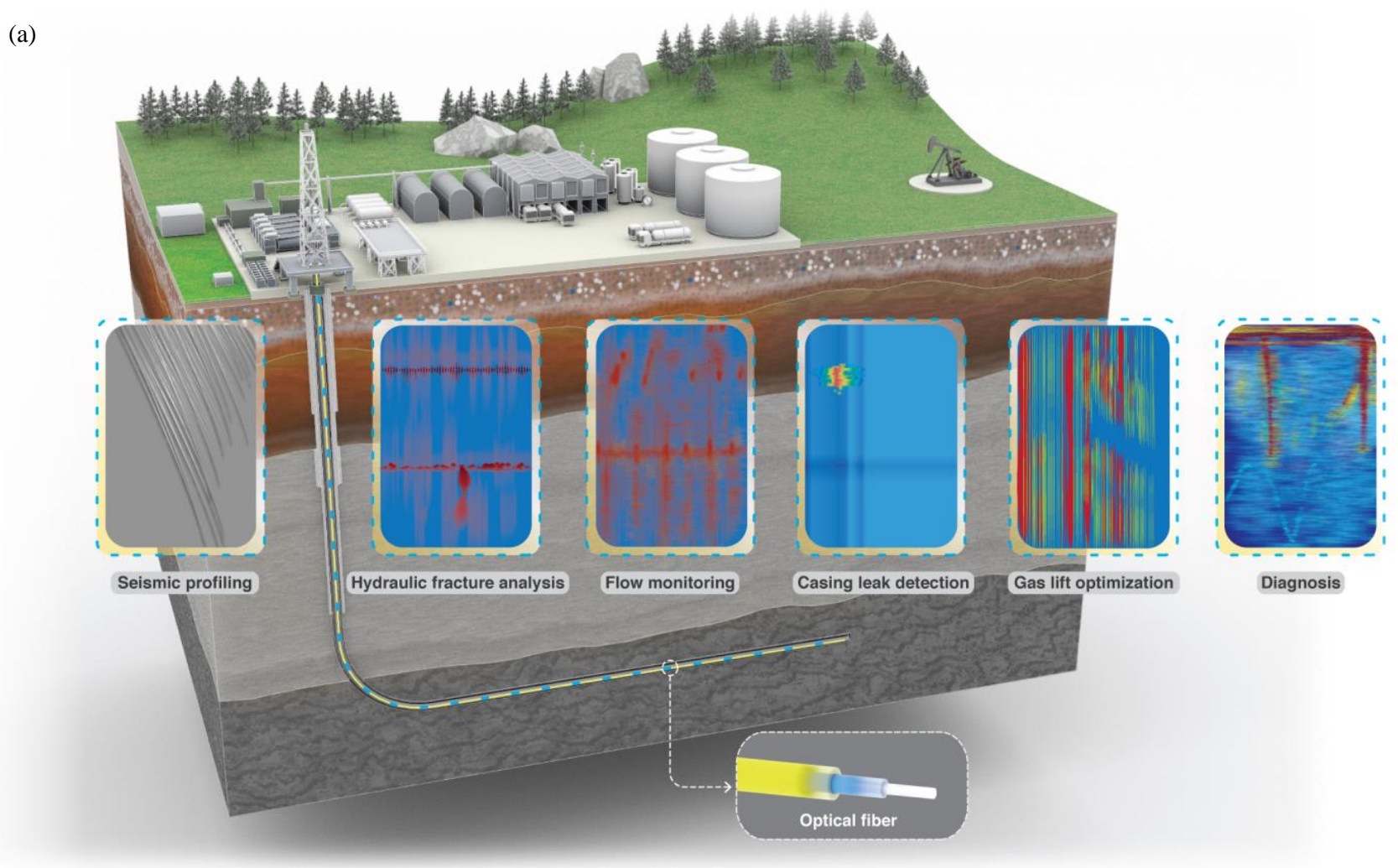

(b)
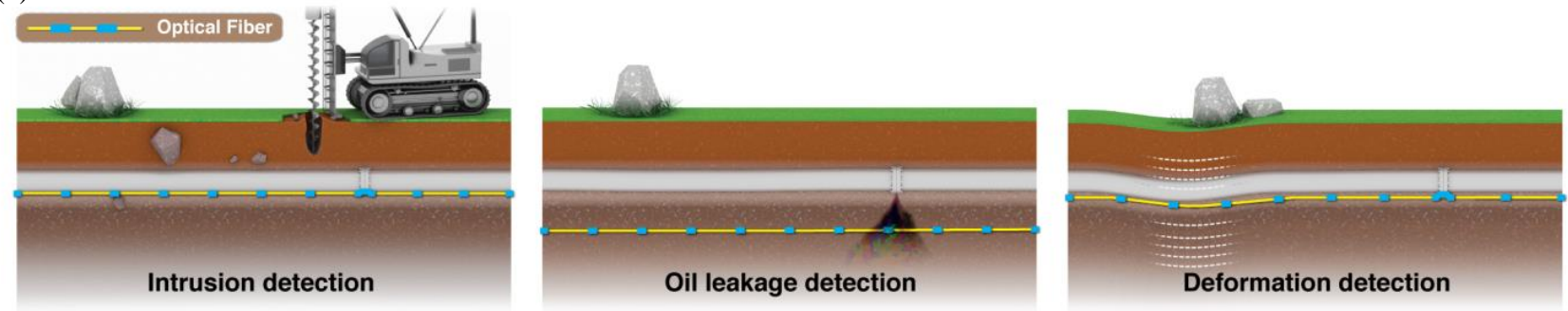

Fig. 2. Examples of distributed fiber-optic sensing applications in the downhole environment (a) and along pipelines (b). OKing Abdullah University of Science and Technology (KAUST).

, time-, and spatial-division multiplexing, arrays of discrete single-point sensors can be multiplexed along the fiber to form a quasi-distributed fiber-optic sensor [19]. In the oil and gas industry, the single-point and quasi-distributed fiber-optic sensors have been deployed in many applications, primarily requiring discrete monitoring of acoustics, temperature, and/or pressure along pipelines or downhole, such as managing well drawdown, in-well pressure measurement to determine completion effectiveness, providing pressure build-up data, zonal production allocation, determination of productivity index, and monitoring during well ramp-up [20]-[22]. However, in terms of technology and cost, single-point and quasi-distributed fiber-optic sensors are not suitable for petroleum applications that require continuous spatial sensing, similar to downhole monitoring of hydrocarbon flow [23], fluid injection [24], wax buildup [23], and surveillance of leak detection along pipelines [25].

In contrast, distributed fiber-optic sensors can offer wealthy information by monitoring environmental parameters along the entire fiber length, i.e., the fiber itself is the sensor [16], [26]. Besides the aforementioned advantages of optical fibers, distributed fiber-optic sensors have another major benefit of reducing the overall sensing cost by measuring sensing parameters continuously and in real-time over tens of kilometers. In the upstream sector, distributed fiber-optic sensors are used for a wide range of applications such as seismic profiling [27], hydraulic fracture analysis [28], flow monitoring [23], casing leak detection [29], gas lift optimization [24], diagnosis [23], among others (Fig. 2(a)). This is achieved by installing optical fibers downhole to deliver data about the well and reservoir. On the other hand, distributed fiber-optic sensors can provide detection of intrusion [30], leak [31], and deformation [32] along a pipeline by attaching/placing the optical fiber to/near the pipeline's surface (Fig. 2(b)).

The operation principles of distributed fiber-optic sensors, widely deployed in the oil and gas industry, are mainly based 
on optical scattering such as Rayleigh, Brillouin, or Raman scattering [33]. Generally, light scattering is a stochastic statistical process that occurs in all angular directions. A typical spectrum of spontaneous light scattering in an optical fiber is shown in Fig. 3, comprising Rayleigh, Brillouin and Raman scattering [16], [26]. During the fabrication process of optical fibers, silica molecules move in the molten state and then randomly freeze in place, which leads to fluctuations in density along the optical fiber. The density fluctuations cause random changes in the refractive index at a scale smaller than the optical wavelength, resulting in Rayleigh scattering. Rayleigh scattering is an elastic phenomenon, i.e., incident light does not transfer energy to the glass and there is no frequency shift between the incident and scattered light. In contrast, Brillouin and Raman scattering are produced by photon-phonon interaction, such that acoustic (optical) phonons are involved in Brillouin (Raman) scattering. Both Brillouin and Raman scattering are inelastic phenomena, in which the frequency of the scattered light is shifted from that of the incident light (Fig. 3). With respect to the central Rayleigh peak, the down- and upshifted spectral components of Brillouin and Raman scattering are called Stokes and Anti-Stokes components, respectively. A last scattering phenomenon that can be observed in Fig. 3 is the Rayleigh-wing scattering, produced by fluctuations in the orientation of anisotropic molecules of the scattering medium [34].

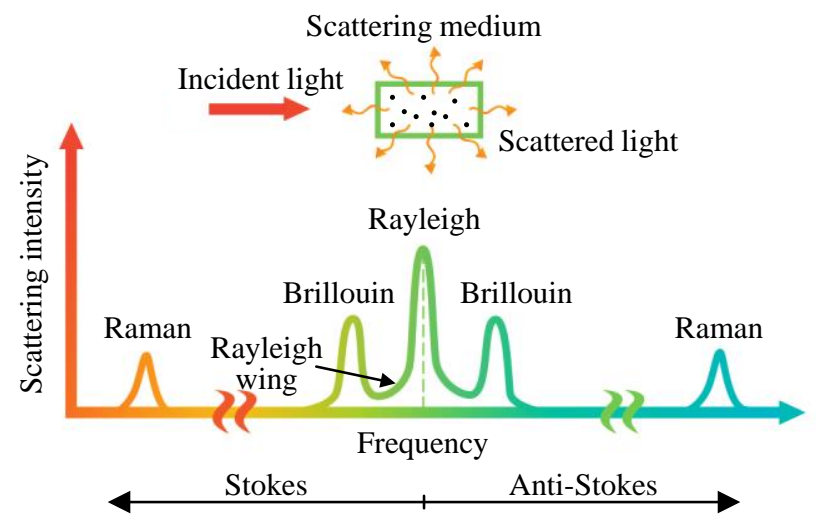

Fig. 3. A typical spectrum of spontaneous light scattering.

Optical scattering can be classified as spontaneous or stimulated, based on the change in the optical properties of the medium during scattering. Spontaneous scattering typically occurs at low levels of incident light intensity, which do not alter the medium's optical properties. As the intensity of the incident light increases to a level at which the light changes the medium's optical properties, the scattering becomes stimulated. In other words, the transition from spontaneous to stimulated scattering corresponds to a change in the behavior of the medium from a linear to a nonlinear optical regime [34].Both spontaneous and stimulated optical scattering offer various capabilities for distributed fiber-optic sensors used in the oil and gas industry and other applications.

In this work, we review the deployment of distributed fiberoptic sensors in the oil and gas industry. The review first focuses on the operation principles and petroleum applications of the fiber-optic Rayleigh DAS and Raman DTS that are widely used in the oil and gas industry. We then describe our design of a multimode fiber (MMF)-based hybrid DAS-DTS system. Using a single interrogation sensing unit in this hybrid system, vibration locations and frequencies, and temperature profile are simultaneously measured on-site in an oil well, for the first time to the best of our knowledge. Next, we briefly highlight the operation principle and petroleum applications of the fiber-optic Brillouin distributed temperature and strain sensing (DTSS), which is less commonly used in the oil and gas industry compared to the DAS and DTS. The review further sheds light on commercially available fiber-optic DAS, DTS, and DTSS products serving the oil and gas industry. Finally, we point out the main challenges that may hinder the growth of the distributed fiber-optic sensor market in the oil and gas industry, and further discuss the potential future directions of that sensing technology.

\section{FIBER-OPTIC DISTRIBUTED ACOUSTIC SENSING (DAS) BASED ON RAYLEIGH SCATTERING}

It is estimated that the oil and gas industry will lead the market of the fiber-optic DAS. The DAS market for the oil and gas sector was valued at USD 129.4 million in 2020 and is expected to reach USD 224.6 million by 2026, at a CAGR of $9.5 \%$ between 2021 and 2026 [35]. In this subsection, we describe the basic physics and operation principle of the fiberoptic DAS, followed by its main applications in the oil and gas industry.

\section{A. Operation principle of the DAS}

The Rayleigh-based fiber-optic DAS is implemented using the phase-sensitive optical time domain reflectometer $(\Phi-$ OTDR), which was initially described in [36]-[39]. The operation principle of the fiber-optic DAS is very similar to the traditional OTDR [16], with a main exception of using a laser source with a narrow linewidth and a stable frequency. In other words, the successful operation of the DAS system is contingent on the use of a laser source with a coherence length much longer than the fiber under test (FUT) used. The majority of commercial fiber-optic DAS systems adopt the direct detection method (Fig. 4(a)), which significantly reduces the complexity of the sensing system [16], [40]. In this setup, a narrow linewidth laser source generates a continuous wave $(\mathrm{CW})$ light. The $\mathrm{CW}$ light is then converted into optical pulses using a modulator, such as electro-optic modulator (EOM) or acousto-optic modulator (AOM), driven by a function generator. Next, an erbium-doped fiber amplifier (EDFA) amplifies the power of the modulated optical pulses, which are launched into a single-mode fiber (SMF) via a circulator. As the optical pulses propagate along the SMF, Rayleigh signals are backscattered to be directed through a circulator toward another EDFA. The EDFA's amplified spontaneous emission (ASE) noise is then discarded using a filter, and finally the filtered Rayleigh signals are detected and recorded respectively using a photodetector (PD) and a data acquisition device (DAQ). 

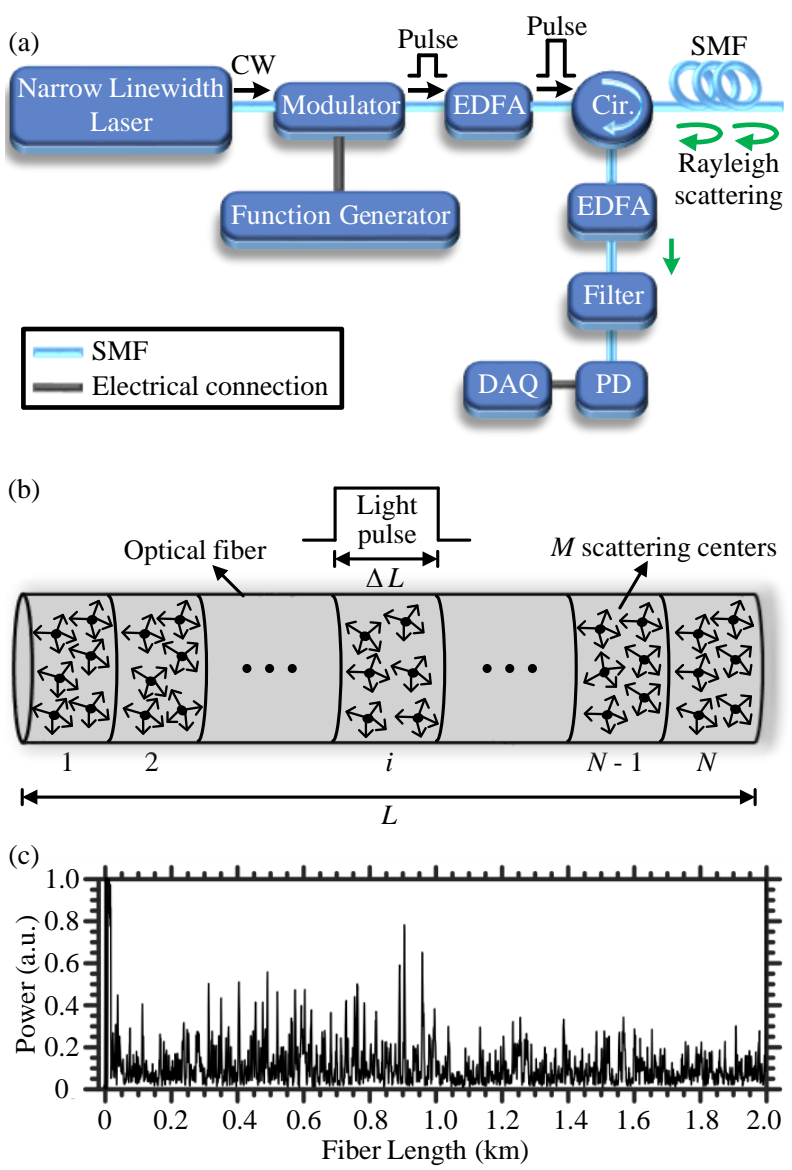

Fig. 4. (a) Experimental setup of the fiber-optic DAS with the direct detection method. (b) Schematic of modeling the operation of the fiberoptic DAS. (c) Representative example of a Rayleigh trace, recorded using a fiber-optic DAS. Cir., circulator.

The operation principle of the fiber-optic DAS can be modeled by dividing the length $(L)$ of the optical fiber into cascaded $N$ small sections, such that each section length $\Delta L=L / N$ (Fig. 4(b)) [41], [42]. In this model, it is reasonable to set $\Delta L$ equals the width of the optical pulse. Due to the density fluctuations along the optical fiber, it is assumed that each section contains $M$ independent Rayleigh scattering centers. At the PD, the interference field $E^{i}$ of the returned Rayleigh light generated from the $i^{\text {th }}$ section can be expressed as:

$$
E^{i}=E_{0} e^{-2 \alpha L_{i}} \sum_{k=1}^{M} r_{k}^{i} e^{j \phi_{k}^{i}},
$$

where $E_{0}$ is the electric field of the incident light, $\alpha$ is the attenuation coefficient of the fiber, $L_{i}$ is the distance between the input port of the optical fiber to the location of the $i^{t h}$ section, i.e., $L_{i}=i \Delta L, i=1,2, \ldots, N$. The scattering coefficient and phase of the $k^{t h}$ scattering center in the $i^{t h}$ section are represented by $r_{k}^{i}$ and $\phi_{k}^{i}$, respectively. Eq. (1) can show that the $i^{t h}$ section's Rayleigh backscattered intensity $I^{i} \propto\left|E^{i}\right|^{2}$ is linked to the relative phases of light reflected from the individual scattering centers within the $i^{t h}$ section. Since the scattering centers are spatially randomly distributed within a section of the fiber, the fiber optic DAS has a Rayleigh speckle-like profile of random intensities along its length, as shown in the representative example of Fig. 4(c) for a $2-\mathrm{km}-$ long standard SMF.

In the absence of intrusion along the FUT, i.e., no refractive index perturbation along the fiber, the recorded Rayleigh traces remain ideally identical in the time-domain. In the case where an acoustic signal is applied at a position along the FUT, the relative phases of the backscattered light at that position change, resulting in temporal intensity fluctuations of the Rayleigh traces only at that perturbation position. The location of the acoustic event along the FUT can be identified by applying the differential method, in which consecutive temporal Rayleigh traces are subtracted from an initial reference one [43]. In addition, the frequency components of the acoustic event can be calculated by applying the fast Fourier transform to the resulting differential signal at the perturbation position.

Although the direct detection method is simple, as shown in Fig. 4(a), it has the drawback that the Rayleigh differential intensity varies nonlinearly with the strain induced by an acoustic event [42]. In other words, the strain along the FUT cannot be properly quantified. In case an application, such as wellbore seismic acquisition, strictly requires quantifying the strain along the FUT, differential phase calculation should be adopted instead of the differential intensity measurement. The differential phase varies linearly with the strain; however, calculating the differential phase requires the use of more sophisticated optical systems and signal processing methods. For example, interferometric recovery [44], coherent detection [45], [46], dual-pulse [36], or chirped-pulse [47] scheme can be used for calculating the differential phase.

As a representative example, we describe the operation principle and experimental setup of the interferometric scheme for recovering the differential phase of a fiber-optic DAS (Fig. 5) [44]. As Fig. 5 shows, the system comprises a distributed feedback (DFB) laser that generates a CW light, which is modulated into optical pulses via an integrated optical chip (IOC). The optical pulses are then amplified with an EDFA, where its ASE noise is filtered out using a tunable FabryPerot filter (TFP). The amplified and filtered optical pulses are then launched throughout a circulator into a sensing fiber, whose distal end is optically terminated (OT) to prevent back reflections. The backscattered Rayleigh light is directed toward an unbalanced Mach-Zehnder interferometer (MZI), which mixes the backscattered light from two separate regions along the sensing fiber to extract the differential phase between them. The optical pulse-width determines the length of the scattering region $\left(L_{P W}\right)$, while the gauge length $(G L)$ is half the interferometer optical path imbalance. To avoid phase signal fading [48], a $3 \times 3$ output coupler is used for the MZI such that the interferometer has a $2 \pi / 3$ relative phase difference between the output arms of the $3 \times 3$ coupler. The three output signals from the MZI are detected with avalanche photodiodes (APDs), sampled with a sample and hold amplifier (S/H), and processed for differential phase extraction along the fiber.

Demodulating the differential phase $\Delta \phi(l)$ for any given fiber section of length $l$ is well explained in [48], [49]. The light intensity at the three output arms of the MZI can be 


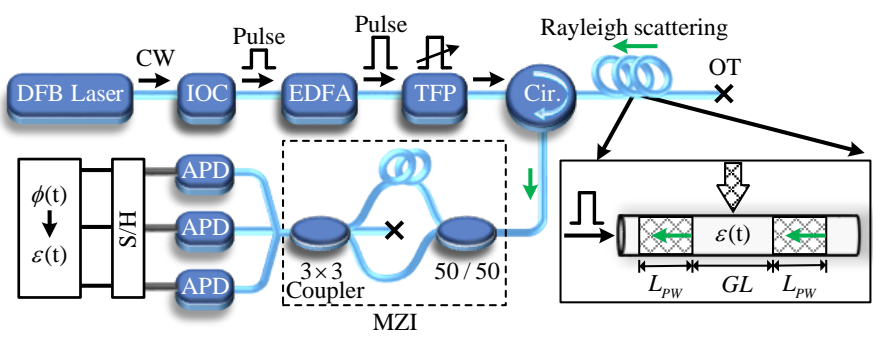

Fig. 5. Experimental setup of the fiber-optic DAS with the interferometric scheme for recovering the differential phase.

expressed as:

$$
\begin{aligned}
& I_{1}=I_{0}[M+N \cos (\Delta \phi(l))], \\
& I_{2}=I_{0}\left[M+N \cos \left(\Delta \phi(l)+\frac{2 \pi}{3}\right)\right], \\
& I_{3}=I_{0}\left[M+N \cos \left(\Delta \phi(l)-\frac{2 \pi}{3}\right)\right],
\end{aligned}
$$

where $I_{0}$ is the intensity of the input signal, and $M$ and $N$ are constants. Using trigonometric identities and some mathematical manipulation, $\Delta \phi(l)$ can be extracted as:

$$
\Delta \phi(l)=\arctan \left(\frac{\bar{I}_{2}-\bar{I}_{3}}{\bar{I}_{1}}\right),
$$

where $\bar{I}_{i}, i=1,2$, or 3 , is the intensity of light at the detector less its DC component. Once $\Delta \phi(l)$ is demodulated, the strain $(\epsilon)$ applied on the fiber section can be estimated using the formula below:

$$
\Delta \phi(l)=\epsilon l \beta \times 0.78,
$$

where $\beta$ is the propagation constant of light in the fiber.

It is important to highlight that the operation of DAS system that measures differential intensity/phase relies on spontaneous Rayleigh scattering. It is recommended to increase the peak power of the short optical pulses used in the fiber-optic DAS to extend the sensing range while maintaining a high spatial resolution. However, the peak power of the pulses should not exceed a threshold beyond which nonlinear phenomena occur within the fiber, such as self-phase modulation (SPM) and modulation instability (MI) which are generally the first nonlinear effects to degrade DAS measurements [50]-[52]. The threshold power of nonlinearity relies on the details of the optical pulses and fiber type.

\section{B. Applications of the DAS in the oil and gas industry}

Although there are many emerging applications for fiberoptic DAS in the oil and gas industry, here we focus on the mature applications.

1) Vertical seismic profiling (VSP): Exploring the seismic activities of underground formations allows scientists to identify the geology of the subsurface layers [53]. Seismic techniques can be categorized as passive or active. In the passive seismic technology, acoustic sensors detect both natural and humanmade microseismic events coming directly from the subsurface and are caused by rock fracturing. In contrast, the active seismic technology involves the generation of artificial sound that propagates through the subsurface and meanwhile, the generated sound is either detected by acoustic sensors inside the well or its reflected waves are recorded by surface sensors. Fiber-optic DAS has been widely used in seismic monitoring applications [54], [55].

(a)

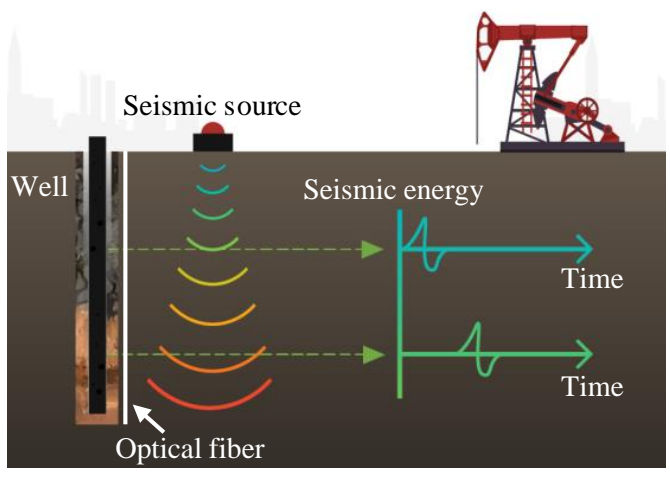

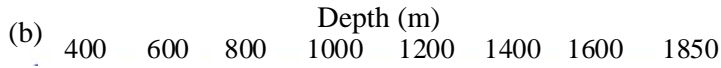
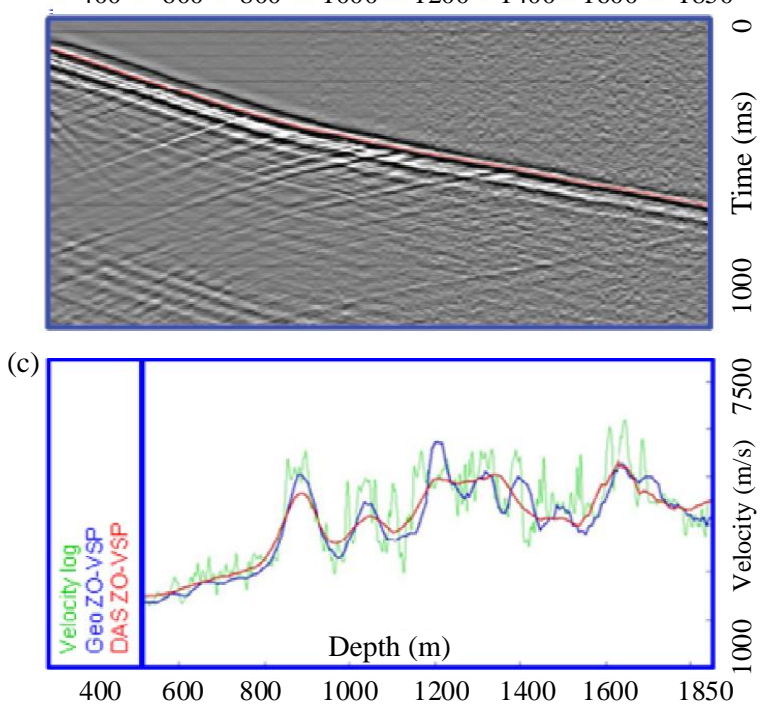

Fig. 6. (a) Acquiring a VSP survey using a fiber-optic DAS. (b) A VSP survey recorded with DAS. (c) Velocity profiles recorded with different sensing systems. ((b) and (c) are reproduced from [54] with permission).

An important application of active seismic techniques is the vertical seismic profiling (VSP) survey, which can be recorded using the fiber-optic DAS system [27] or geophone arrays [56]. Geophones produce VSP survey at discrete locations; thus covering the entire well requires several movements to the geophone arrays. In addition, geophones often cannot be deployed in production wells. In contrast, fiber-optic DAS is a game-changer for downhole seismic acquisition as it can produce the VSP survey along the entire well at once and permanently, even for producing wells.

The typical technique of acquiring an DAS-based VSP survey is shown in Fig. 6(a) [12], [15]. A seismic source is actively generated on the surface, while the fiber-optic DAS records the propagating acoustic signal downhole. By tracking the arrival times of the downgoing seismic energy, one can accurately determine the seismic velocity along the vertical direction. The seismic velocity determined from the 
VSP survey can estimate, for example, the composition of subsurface materials such as the oil-to-water ratio [24]. A limitation of the VSP survey produced by the fiber-optic DAS is that it provides an estimate of the subsurface properties in the proximity of the optical fiber, which is a drawback of the geophone arrays as well. However, the use of fiber-optic DAS for time-lapse (also known as 4-D) VSP can monitor the movement of fluids on a reservoir scale [57].

As a representative example, Fig. 6(b) shows a VSP survey recorded using a fiber-optic DAS in a project for storing and monitoring $\mathrm{CO}_{2}$ underground. As Fig. 6(b) shows, the first arrival picks (red line) are strong enough to calculate the velocity profile. Using the derivative in depth of the picked first arrival times, one can obtain the velocity profile (Fig. 6(c)) and thus help monitor $\mathrm{CO}_{2}$ containment. As shown in Fig. 6(c), the velocity calculated using the fiber-optic DAS (red line) is generally in good agreement with those calculated with a geophone (blue line) and a sonic log (green line). However, the DAS velocity profile is smoother due to the unique distributed sensing feature of DAS.

A technical challenge for the proper operation of fiberoptic DAS, especially in seismic monitoring applications, is that the acoustic energy should be efficiently transferred to the fiber-optic cable, which requires strong coupling between the medium and the cable. Traditional techniques of permanent fiber deployment downhole for DAS include locating the fiber cable on casing, on tubing, or inside tubing (Fig. 7). Fig. 7 further compares the data quality, cost, removability, and effective depth of the three traditional types of deployment. To improve the overall performance of fiber deployment downhole for DAS system, flexible borehole liners can be used to couple the fiber-optic cable against the borehole wall (Fig. 7) [58]. This technology can improve the SNR of the fiber-optic DAS in various applications; however, it suffers from the depth limitation. on the other hand, especially during early reservoir evaluation, optical fiber cable can be introduced on intervention devices, such as a wireline cable [6], [7]. One of the challenges for the fiber deployment with wireline is to ensure adequate coupling to the borehole or casing especially for vertical wells, but the coupling can be improved by giving a slack to the wire cable.

Another way to improve the performance of DAS is to use engineered optical fiber, which enhances backscattered Rayleigh light [27], [30]. Although the engineered fiber can enhance the SNR of DAS, it is likely to increase the optical attenuation of the pump pulses which may degrade the sensitivity of DAS over long distances. One optimum technique for the use of the engineered optical fiber is it to splice/join it with the distal end of a standard SMF, where the backscattered Rayleigh signal's intensity starts to degrade. In general, compared to standard optical fibers, engineered optical fibers are more expensive and may not be flexible to deploy with typical DAS interrogation units. Thus, standard SMF is the preferred platform for DAS unless an application requires extending the sensing range over a longer distances where engineered fiber can be combined with the standard one.

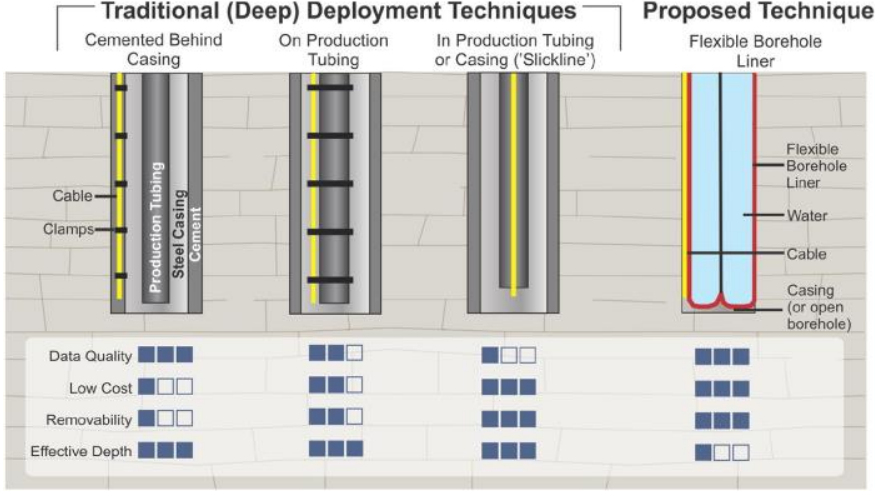

Fig. 7. Comparison of downhole fiber deployment techniques for DAS system. (Reproduced from [58] with permission).

2) Hydraulic fracturing monitoring: In some reservoirs, hydrocarbons can be deeply trapped in tight formations. To release the hydrocarbons and allow them to flow into the wellbore, the solid formation has to be fractured. Hydraulic fracturing is a well-stimulation method used to increase the flow of hydrocarbons into the well from oil-bearing rock formations (Fig. 8(a)) [59]. The hydraulic fracturing typically comprises injecting proppant, water, and chemicals under a high pressure into the tight formation through the well. This process aims to create new fractures in the rock as well as enlarging existing fractures, facilitating the flow of hydrocarbons into the production well. It is highly beneficial to monitor the development of fractures that occur during and after hydraulic fracturing. Production engineers can take advantage of this monitoring data to understand the nature of the induced fracture level in different areas. In addition, monitoring the hydraulic fracturing can lead to locating the source of hydrocarbons within the reservoir [15].

Hydraulic fracturing monitoring is one of the main applications of the fiber-optic DAS [55]. In real-time, a fiberoptic DAS can locate where fractures begin, determine the effectiveness of the stimulation process, and optimize the placement of fluid and proppant. Following the initiation of the hydraulic fracturing, fiber-optic DAS can detect the acoustic energy generated by hydrocarbons as they flow through the fractures. For example, in a certain region if there is no or weak acoustic energy is detected, this indicates no sufficient fluid and proppant were delivered to this region.

As a representative example, we present a recording of a hydraulic fracturing process using a low-frequency $(0-0.5 \mathrm{~Hz})$ DAS (LF-DAS) [28]. In an observation well, an optical fiber cable is installed behind the casing. The observation well is $200 \mathrm{~m}$ from the operating well, where hydraulic fracturing is applied. The fracturing is carried out in the horizontal section of the operation well from the well's toe to heel. As preprocessing steps for the LF-DAS, the DAS data were first downsampled from $4 \mathrm{kHz}$ to $1 \mathrm{~Hz}$ with an antialiasing filter $(0-0.5 \mathrm{~Hz})$ to extract the low-frequency band. Then, to reduce noise, a median filter and DC removal filter were applied. The DAS interrogation unit measures a response proportional to the strain rate observed along the well (Fig. 8(b)). To clarify, 

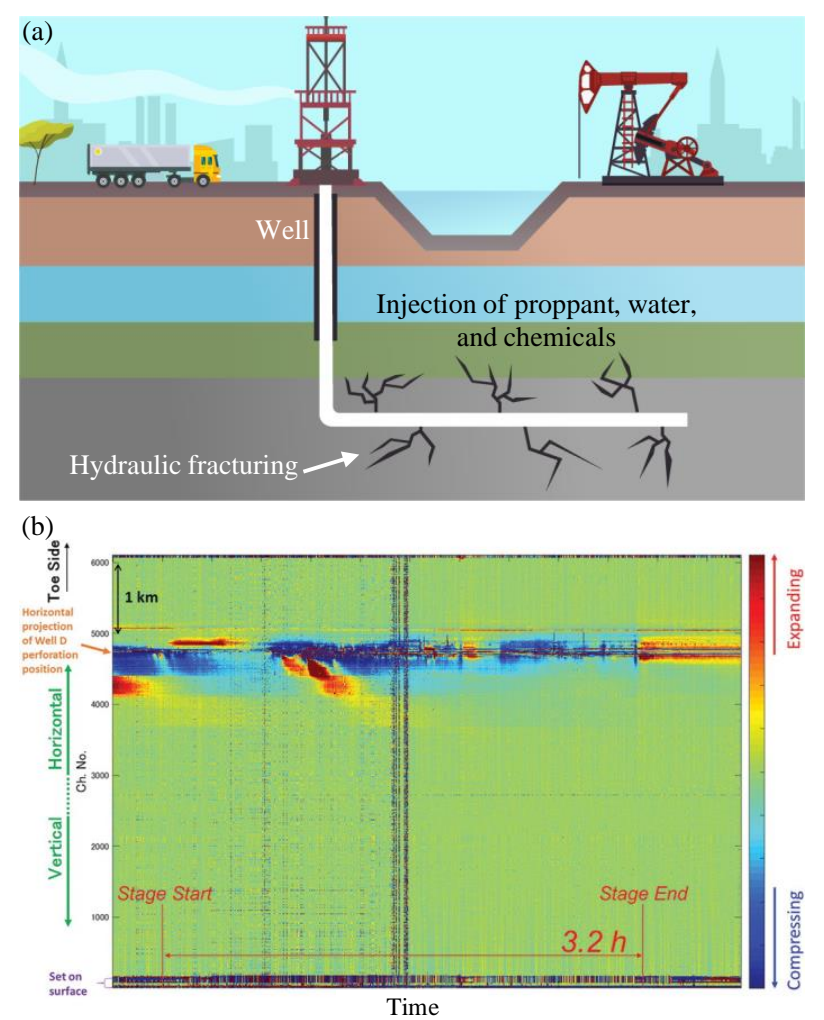

Fig. 8. (a) Schematic image of the downhole hydraulic fracturing. (b) A recording of a hydraulic fracturing process using a LF-DAS. (b) is reproduced from [28] with permission).

regarding the colormap of Fig. 8(b), the red/blue response indicates expanding/compressing strain rate.

As shown in Fig. 8(b), clear responses can be observed around the perforated section (around channel No. 4700 along the vertical axis), while other sections are very quiet. The expanding points shown (red lines around channel No. 4700) are closely correlated to the parallel projected perforation points. The red color converted to blue immediately following the end of the operation. These observation results indicate that the responses observed in Fig. 8(b) were closely related to the fracturing operation. It is important to highlight that the low-frequency $(<2 \mathrm{~Hz})$ DAS is cross-sensitive to strain and temperature perturbations [60]. Hence in [28], another optical fiber cable was installed for DTS measurement to further understand the temperature changes around the compression response near the fracture hit position and time.

3) Downhole flow monitoring: In-well flow monitoring is an essential process in the oil and gas industry to enhance production and maintain safety requirements. The contributions of fiber-optic DAS to flow monitoring include [23], [24]: 1) zonal injection/production profiling across inflow control valves (ICVs), 2) detection of interzonal inflow from the reservoir, 3) monitoring injection from gas-lift valves (GLVs), 4) tracing fluid movement through the borehole, and 5) observing wax build-up.

Fig. 9 shows some of using a DAS optical fiber to monitor a downhole flow [23]. The figure represents a 30-min recording of a DAS from an oil producer, which includes GLVs and ICVs where their locations are shown in the completion diagram of Fig. 9. The repetition frequency and spatial resolution used in this measurement were $10 \mathrm{kHz}$ and $10 \mathrm{~m}$, respectively, while the DAS data were processed to preserve only the acoustic information in a medium-frequency range. This frequency range works well for determining the flow from ICVs and GLVs. The acoustic information in this frequency band is displayed in Fig. 9 using an image where blue color is used to indicate quiet regions of the well (low flow) and yellow to red colors indicate increasingly loud events in the well (high flow). The results of Fig. 9 can clearly describe the operating states of the GLVs and ICVs: only the ICVs B and D are opened, while the remaining ICVs and all the GLVs are closed. Besides, near the wellhead, a broad acoustic event is recorded in the DAS data. It was confirmed that this acoustic energy is generated by a build-up of wax, as there is no completion element in this region and this producer was known to be a waxy well. Wax build-up within the tubing producing the hydrocarbon results in a blockage of the producer well, which creates acoustic noise.

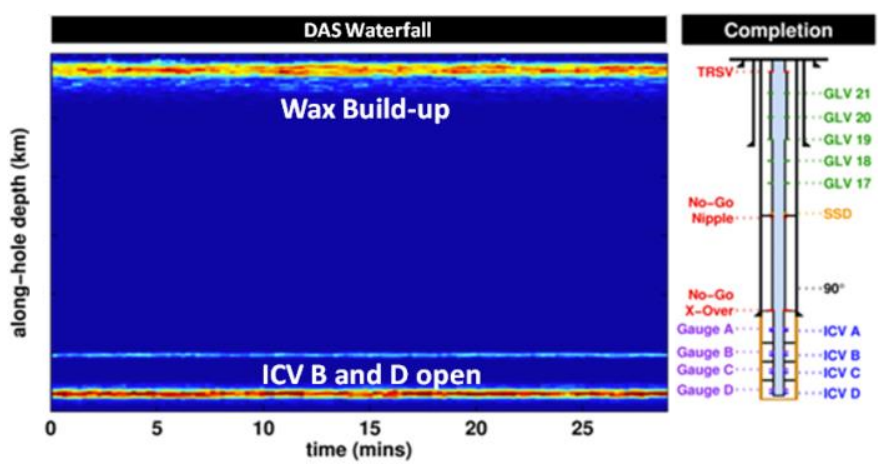

Fig. 9. Fiber-optic DAS data of a smart producer with a wax build-up. (Reproduced from [23] with permission).

4) Well integrity: Well integrity assessment, such as detecting leaks through casing within a wellbore, is challenging using the geophone arrays. This is mainly because geophones cannot cover the entire well at once, resulting in a timeconsuming process of moving the geophones along the well. In contrast, fiber-optic DAS allows the determination of the depth of a leak or several leaks, with a full-coverage to the wellbore [29], [61]. In addition, DAS can provide a gas movement profile caused by a casing failure, a wellhead seals failure, etc.

Fig. 10 shows an example of using a fiber-optic DAS to detect a gas leak event in a water-filled abandoned vertical conventional gas well [29]. The colormap of Fig. 10 represents the changes in acoustic amplitude within a $(50-100 \mathrm{~Hz})$ frequency band, where the acoustic noise produced by the leak is evident. After the surface casing vent of the well was opened, a continuous acoustic event was recorded at a depth of $275 \mathrm{~m}$ (around channel No. 234 in the vertical axis of Fig. 10). The strength of this event gradually decreased after the vent closed. A noise logging tool (hydrophone) confirmed the presence of the acoustic event at the same location. Based on the results collected using the DAS and hydrophone, it is 
expected that a casing leak is indeed at the 275-m depth. This discussion highlights the advantages of the DAS system over hydrophone: the detection of this leak event would be missed, if no hydrophones were present at the event's location when it occurred.

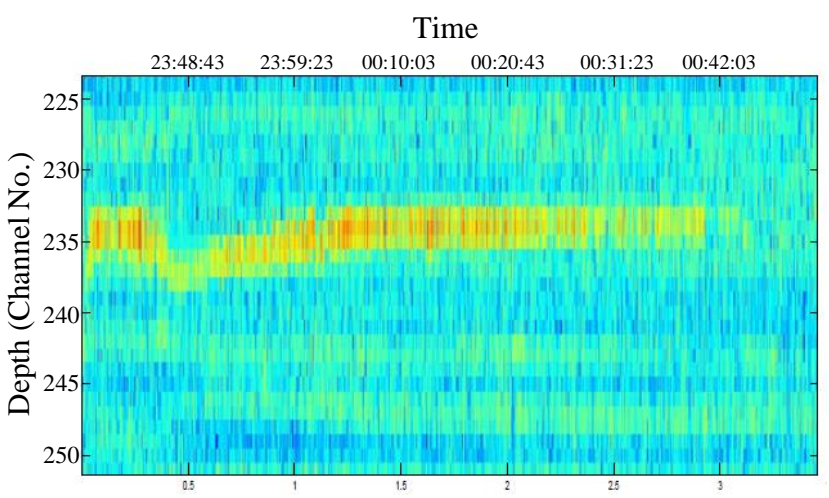

Fig. 10. Casing leak detection using a fiber-optic DAS. (Reproduced from [29] with permission).

5) Pipeline intrusion detection: In the oil and gas industry, the midstream and downstream sectors rely on vast networks of pipelines to transport hydrocarbons to refineries and retail distributors. It is important to establish a comprehensive surveillance system to protect pipeline integrity from external threats, such as hydrocarbon theft, accidents, etc. Compared to video surveillance and manned monitoring, fiber-optic DAS can offer pipeline intrusion detection over much longer distances. Using machine learning algorithms to process the data of the fiber-optic DAS, various activities near the pipeline, such as vehicular traffic, digging, and human walking, can be classified [62].

Fig. 11 is a representative example of a DAS-based system used to monitor a pipeline [30]. In this experiment, a speciality optical fiber with an enhanced Rayleigh scattering is used to improve the detection sensitivity of the DAS system. This is achieved by using a focused femtosecond laser to produce permanent nano-grating points in the core of a standard telecommunication SMF. The laser-induced Rayleigh scattering points are wavelength independent and stable at high temperatures. As shown in Fig. 11, there are seven fiber sensors along the pipeline, with each sensor comprising at least one laserinduced Rayleigh scattering point. The interrogation unit of the DAS system (inset Fig. 11) extracts the differential phase, and thus the DAS does not suffer from the aforementioned nonlinear behavior of the direct detection system. Four types of hammer heads (soft rubber, hard plastic, aluminum, and steel) are used to generate extrinsic acoustic signals that mimic different potential pipeline intrusion events. A machine learning algorithm combined with the DAS achieves an accuracy of more than $85 \%$ for identifying the different external intrusion events.

The system presented in [30] can generally be considered as distributed sensing because the fiber used is standard SMF with laser-induced Rayleigh enhancement at discrete points. In other words, the DAS system can be operated using either

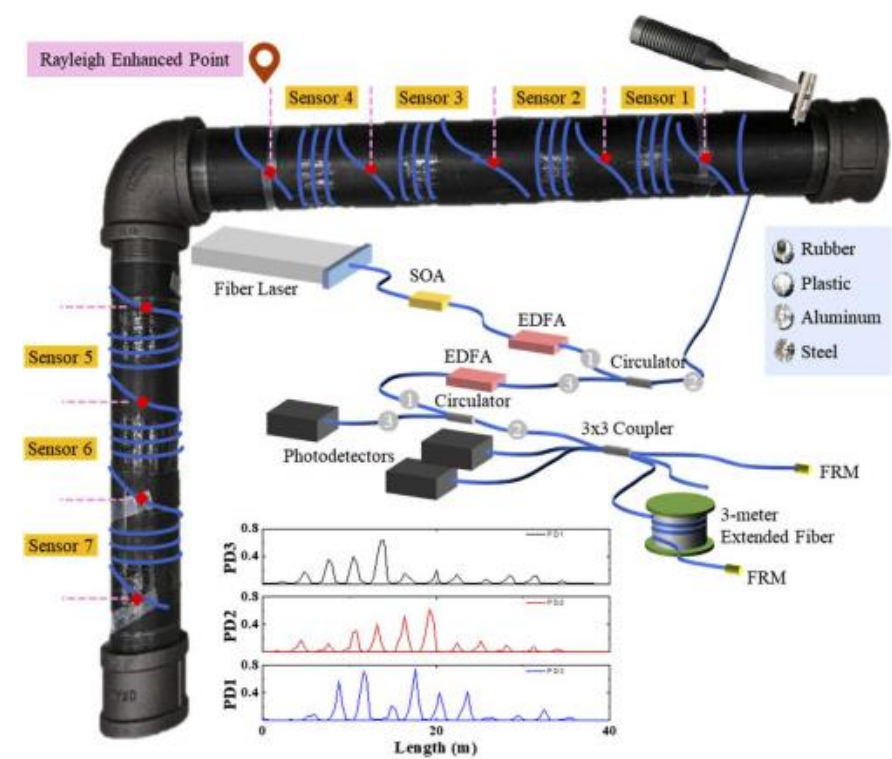

Fig. 11. A pipeline intrusion detection system using a fiber-optic DAS and a machine learning algorithm. (Reproduced from [30] with permission).

the typical or enhanced Rayleigh scattering. However, this DAS-based pipeline monitoring technique relies on processing the Rayleigh scattering from the nano-grating points that are separated by $\sim 3-\mathrm{m}$ fiber sections and meanwhile the system has a $\sim 1-\mathrm{m}$ spatial resolution. Given this design, the monitoring system can be considered as an example of a quasi-distributed sensor. Another drawback of this approach is that for different pipeline configurations and shapes, the neural networks used are likely to be retrained.

\section{FIBER-OPTIC DISTRIBUTED TEMPERATURE SENSING (DTS) BASED ON RAMAN SCATTERING}

In the oil and gas industry, monitoring temperature is critical for upstream, midstream, and downstream applications. Compared with Brillouin-based distributed fiber-optic sensors that are sensitive to temperature and strain, Ramanbased distributed fiber-optic DTS is almost only temperaturesensitive [16]. This unique near zero cross-sensitivity feature of Raman-based DTS makes it the preferred platform for distributed temperature sensing. The oil and gas industry is anticipated to lead the market of the Raman-based fiberoptic DTS. The Raman-based DTS market for the oil and gas industry was valued at USD 223 million in 2020 and is expected to reach USD 361 million by 2026 , at a CAGR of $8.3 \%$ between 2021 and 2026 [35].

\section{A. Operation principle of the Raman-based DTS}

The concept of DTS using Raman scattering was first introduced in 1984 [63]. Commercially available Ramanbased DTS systems typically use Raman intensity variations to determine the temperature profile along an optical fiber. The low power of the Stokes and Anti-Stokes Raman signals (60-70 dB weaker than the input pump power) is considered an early obstacle to the deployment of Raman-based DTS 
[64]. However, with the development of high-power lasers, EDFAs, high-sensitivity APD, and signal processing (e.g., averaging and wavelet denoising), the signal-to-noise ratio (SNR) of the Raman-based DTS has been significantly improved.

A typical experimental setup of the Raman-based fiberoptic DTS is schematically shown in Fig. 12(a) [65]. A highpower laser generates a $\mathrm{CW}$ light that is modulated into optical pulses using an AOM. The power of the optical pulses are then amplified with an EDFA, to improve the SNR of the DTS system. The EDFA is followed by a $3 \times 1$ wavelength division multiplexer/demultiplexr (MUX/DEMUX). One side of the wavelength division MUX/DEMUX includes three ports (Port 1-3) for adding/dropping signals of wavelengths matching the laser, Stokes Raman, and Anti-Stokes Raman signal, respectively. The other side of the wavelength division MUX/DEMUX includes a common port (Port 4) that simultaneously supports propagating the laser, Stokes Raman, and Anti-Stokes Raman signal. The amplified optical pulses are delivered to a MMF such that the pulses are directed from Port 1 to Port 4 of the wavelength division MUX/DEMUX. As the optical pulses propagate along the MMF, Stokes and Anti-Stokes Raman signals are backscattered, as shown in Fig. 12(a). In the backward direction, the two backscattered Raman signals can be well separated via the wavelength division MUX/DEMUX, detected using two separate APDs, and finally recorded with a DAQ. Figs. 12(b) and 12(c) show respectively representative examples of Anti-Stokes and Stokes Raman signal recorded using a standard 50/125 $\mu \mathrm{m}$ MMF, entirely placed at room temperature.

At the APDs, the received Raman Stokes power $P_{S}(z)$ and anti-Stokes power $P_{A S}(z)$ from a position $z$ along the fiber can be expressed as [16]:

$$
\begin{aligned}
P_{S}(z) & =R_{S}(z) e^{-\left(\alpha_{p}+\alpha_{S}\right) z} P_{0}, \\
P_{A S}(z) & =R_{A S}(z) e^{-\left(\alpha_{p}+\alpha_{A S}\right) z} P_{0},
\end{aligned}
$$

where $R_{S}(z)$ and $R_{A S}(z)$ are respectively the Stokes and anti-Stokes scattering coefficients at the position $z$, the attenuation coefficients of the pump, Stokes and Anti-Stokes light are denoted as $\alpha_{p}, \alpha_{S}$, and $\alpha_{A S}$, respectively, and $P_{0}$ represents the laser power. As described by the BoseEinstein statistics, $R_{S}(z)$ and $R_{A S}(z)$ are proportional to their differential cross sections as follows [66]:

$$
\begin{gathered}
R_{S} \propto \frac{d \sigma_{S}}{d \Omega} \cong \frac{1}{\lambda_{S}^{4}} \frac{1}{1-\exp \left[-\frac{h c \Delta \nu}{K_{B} T(z)}\right]}, \\
R_{A S} \propto \frac{d \sigma_{A S}}{d \Omega} \cong \frac{1}{\lambda_{A S}^{4}} \frac{1}{\exp \left[\frac{h c \Delta \nu}{K_{B} T(z)}\right]-1},
\end{gathered}
$$

where $\lambda_{S}$ and $\lambda_{A S}$ denote respectively the wavelengths of the Stokes and anti-Stokes light, $h$ is the Planck's constant, $c$ represents the speed of light in vacuum, $K_{B}$ is the Boltzmann constant, $\Delta \nu$ is the Raman shift, and $T$ is the temperature. The ratio $R(z)$ of the two backscattered Raman powers is independent of the laser power or the optical coupling
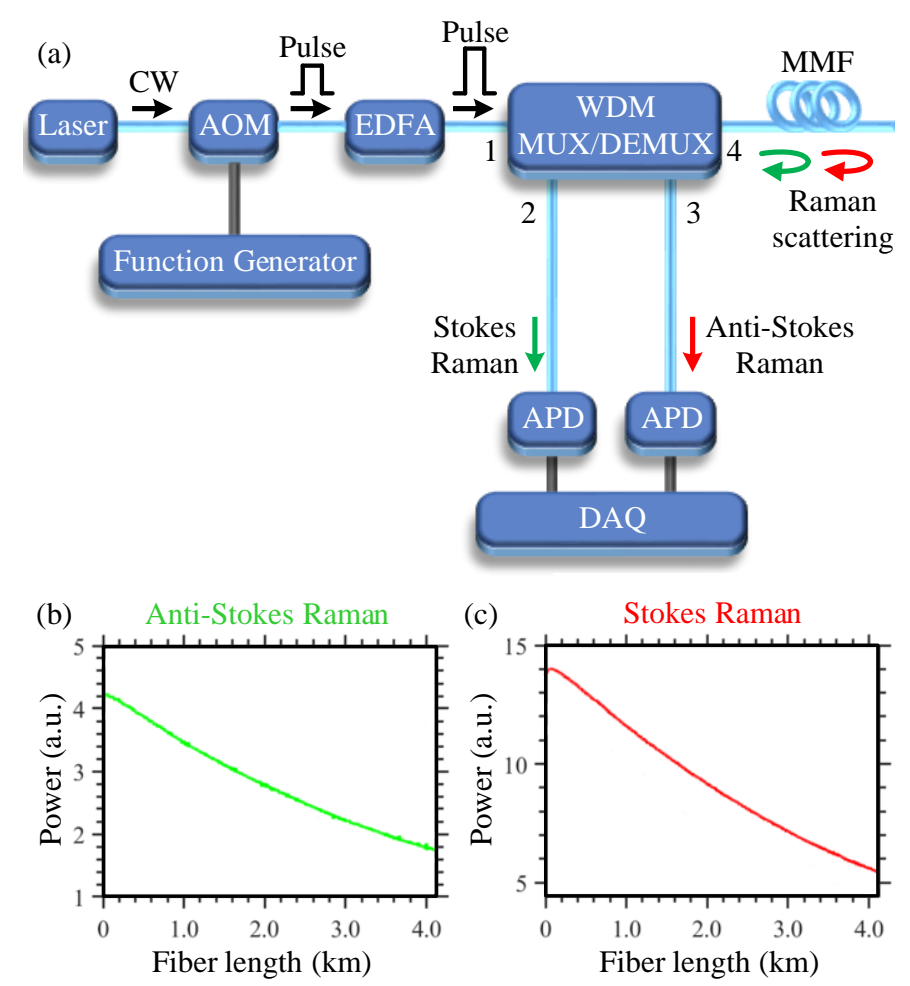

Fig. 12. (a) Experimental setup of a Raman-based fiber-optic DTS system. Representative examples of Anti-Stokes (b) and Stokes (c) Raman signal, recorded at room temperature.

efficiency:

$$
\begin{aligned}
R(z) & =\frac{P_{A S}(z)}{P_{S}(z)} \\
& =\left(\frac{\lambda_{A S}}{\lambda_{S}}\right)^{4} \exp \left(-\alpha_{A S} z+\alpha_{S} z\right) \exp \left(-\frac{h c \Delta \nu}{K_{B} T(z)}\right) .
\end{aligned}
$$

In practical applications, the stability of the DTS system's components may change with the environment. Hence, one usually does not use Eq. 9 directly to calculate the temperature, but instead a known reference temperature $T\left(z_{0}\right)$ at a position $z_{0}$ is introduced as follows:

$$
\begin{aligned}
\frac{1}{T(z)}-\frac{1}{T\left(z_{0}\right)}=\frac{-K_{B}}{h c \Delta \nu} & {\left[\ln \frac{P_{A S}(z)}{P_{S}(z)}-\ln \frac{P_{A S}\left(z_{0}\right)}{P_{S}\left(z_{0}\right)}\right.} \\
& \left.+\left(\alpha_{A S}-\alpha_{S}\right)\left(z-z_{0}\right)\right] .
\end{aligned}
$$

Eq. 10 can be used for determining the temperature $T(z)$ as long as the sensor operates in the spontaneous Raman scattering regime. Stimulated Raman scattering does not follow the Bose-Einstein statistics and cannot be used for distributed temperature sensing. Since the Raman scattering is weak and considering Eqs. 5 and 6 , it is necessary to increase the power of the pump laser to collect a sufficient number of Raman photons for measurement. However, the laser power in DTS cannot exceed the Stimulated Raman scattering threshold, which is proportional to the effective core area of the fiber [67]. 
In the harsh downhole environment of the oil and gas industry, the optical fiber cable may be pumped, exposed to hydrogen or water, etc. Thus, in Eq. 10, the wavelength-dependent differential attenuation coefficient, $\Delta \alpha=\alpha_{A S}-\alpha_{S}$, may change over time and/or along the optical fiber cable. Using a fixed pre-calibrated value of $\Delta \alpha$ cannot produce accurate temperatures over time. Consequently, for temperature measurement downhole, it is necessary to periodically calibrate $\Delta \alpha$ or even calculate it before each measurement. A singleended fiber configuration with a reference temperature sensor at the fiber end or a partially returned fiber configuration can be used to calibrate $\Delta \alpha$, which varies over time but independent of $z$. Otherwise, a double-ended fiber configuration can calibrate $\Delta \alpha$, even if $\Delta \alpha$ changes along the fiber and over time. More details on calibrating the $\Delta \alpha$ of fiber-optic DTS system can be found in [68], [69].

\section{B. Applications of the Raman-based DTS in the oil and gas industry}

Measuring distributed temperature in the downhole environment and along pipelines has many applications. Here, we highlight the important applications of the Raman-based DTS in the oil and gas industry.

1) Downhole flow monitoring: Before production begins in a well, the shut-in temperature profile represents the geothermal temperature, provided that there are no temperature variations because of activities (e.g., mud-circulation, fluid injection, and hydraulic fracturing). Once production begins, the reference geothermal temperature may change significantly at the inflow points due to the flow of hydrocarbons into the wellbore [70].

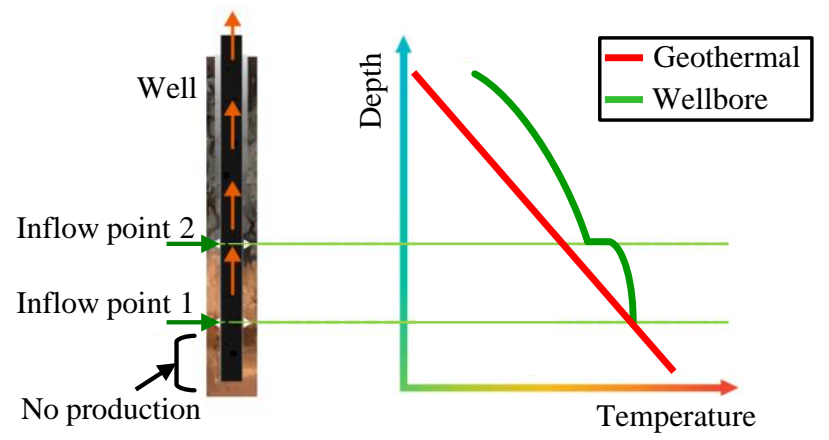

Fig. 13. Locating the inflow points in a wellbore using the Ramanbased fiber-optic DTS.

Fig. 13 shows a representative example for locating the inflow points in a wellbore, using a temperature profile that can be produced with the Raman-based DTS [15]. The geothermal temperature (red line) constantly increases with depth. Focusing on the wellbore temperature profile (green line), where its temperature matches the geothermal gradient at the bottom of the wellbore because there is no production in this region. In this representative example, at the inflow point 1 , fluid enters the wellbore with a temperature corresponding to the local geothermal temperature. As the fluid rises toward the wellhead, heat is transferred between the fluid and the wellbore so that the fluid loses some of its heat to warm the wellbore. At the inflow point 2 , the fluid enters the wellbore again with the new local geothermal temperature, which causes the temperature in the wellbore to decrease due to the fluid mixture. If a gas enters the wellbore, on the contrary, a cooling effect will occur due to adiabatic expansion [15]. Thus, downhole thermal monitoring with the Raman-based DTS can identify inflow points where hydrocarbons enter the wellbore.

2) Injection monitoring: After drilling a production well, through which hydrocarbons flow from the reservoir to the surface, the original pressure of the reservoir is typically sufficient to push the hydrocarbons upward. As the reservoir is producing, however, the original pressure gradually decreases, which requires injection of water into the reservoir to increase its pressure (Fig. 14(a)) [71]. The water injection process shifts the temperature of the hydrocarbons downhole. One of the key factors in the success of the injection process is the identification of the regions where the injection water is transferred to the reservoir.

Downhole temperature measurement recorded by the Raman-based DTS can be used to monitor the performance of injection wells via the warm-back approach. In this approach, after the water injection process, the well is shut in and the well's temperature is observed as it recovers toward the geothermal gradient [68], [72]. Considering the representative example in Fig. 14(b), where a cold water injection changes the temperature of all the surrounding rocks around the well and in the reservoir (purple line). Once the injection is stopped and the well is shut in, the temperature of the rocks gradually returns to the geothermal gradient over time (green line). The temperature of the zones that have absorbed more volumes of water return to the geothermal gradient at a slower rate. Some methods can be applied to allocate the total injected water volumes into each reservoir zone, using temperature surveys [73]. Hence, DTS can also be used to identify outof-zone injection where an unwanted geological layer is pressurized instead of the oil producing layer. This is important in order to execute the injection strategy properly. Also, part of the challenge with injection wells is that the increased pressure might cause integrity challenges in the overburden pipe sections. These wells can also cause integrity issues, with the potential consequence of leaking fluid into shallow water aquifers. Hence, it is environmentally important to monitor some key wells to avoid this potential issue.

3) Steam-Assisted Gravity Drainage (SAGD) Optimization: One technique for the oil recovery enhancement is the steamassisted gravity drainage (SAGD) in which, steam is injected into the reservoir to reduce the viscosity of heavy oil [74]. A SAGD well typically includes a pair of wells, such that a horizontal production well is located approximately $5 \mathrm{~m}$ below another horizontal steam injection well (Fig. 15(a)) [15]. Steam is initially injected into the reservoir through the injection and production wells until forming a sufficient steam chamber, then the producer well is set to a production phase. Due to gravity, the heated heavy oil falls towards the production well and begins to flow to the surface. Meanwhile, the injection well continues the steam injection process to compensate for the heat loss that occurs during the production process. 
(a) Water injection

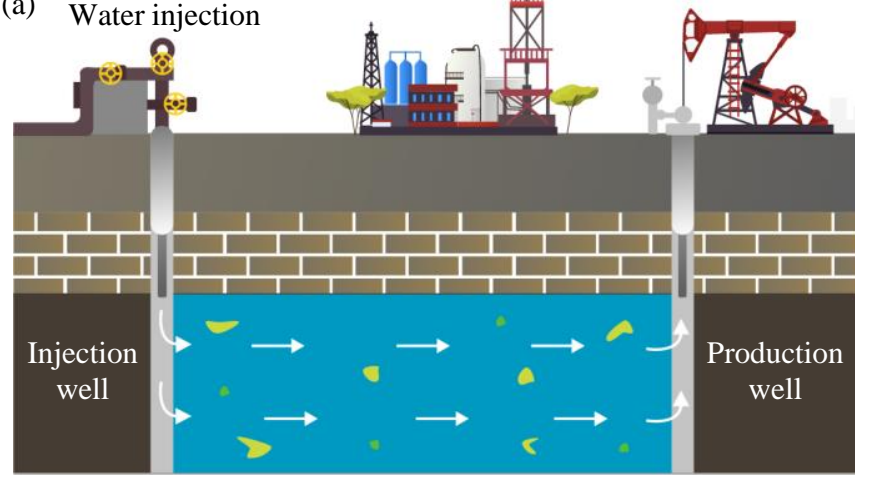

(b)

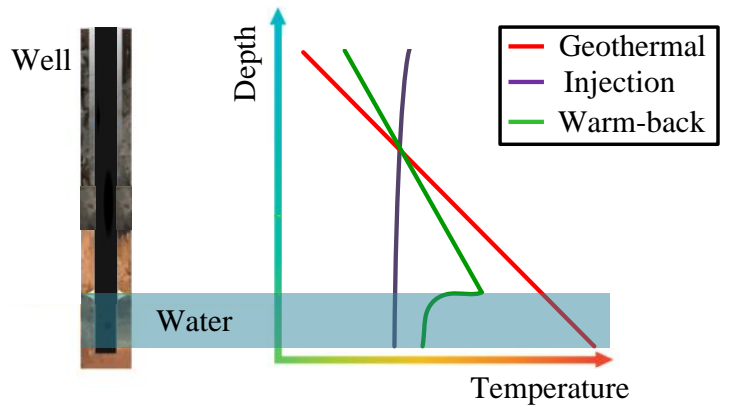

Fig. 14. (a) Schematic image of water injection through an injection well. (b) Warm-back measurement in a water-injection well.

(a)

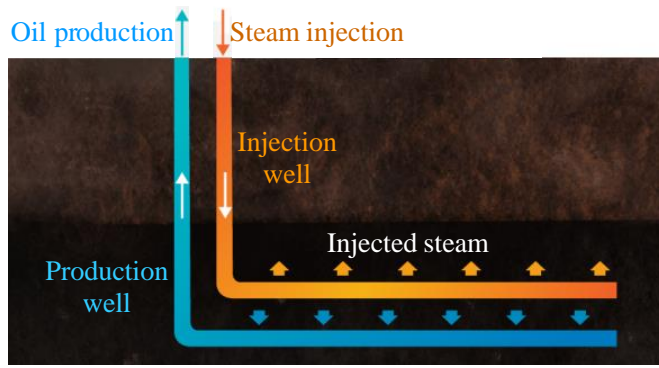

(b)

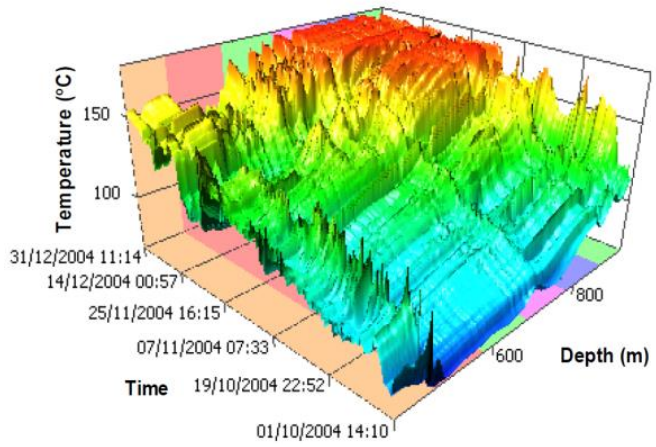

Fig. 15. (a) Illustration showing the SAGD operation using the injection-production well pair. (b) Three-dimensional temperature profile, recorded with a fiber-optic DTS system during an SAGD process. (b) is reproduced from [75] with permission).
Temperature profiles offered by the Raman-based DTS are essential for optimizing the SAGD process [75]. For example, the temperature difference between the injection and production well is a critical parameter that should be monitored. In case the temperature of the production well matches that of the injection well, this is an indication of steam breakthrough into the production well. As a result, the production well will pump the steam/condensed water to the wellhead instead of hydrocarbons. Adjusting the temperature difference between the two wells to an optimum value can improve the SAGD process. Additionally, the temperature of the injector-producer region, which determines the heavy oil viscosity, is considered the main parameter that controls the flow of heavy oil into the production well. This discussion highlights the benefits of using the fiber-optic DTS to improve the SAGD process. However, the temperatures of steam injection are typically well over $200{ }^{\circ} \mathrm{C}$. Due to these high temperatures and harsh downhole environmental conditions, optical fiber cable can suffer degradation because of, for example, hydrogen darkening [76]. Additionally, proper fiber deployment is another challenge for monitoring the SAGD process.

Using a fiber-optic DTS system, Fig. 15(b) shows an example of a temperature profile measurement in an SAGD well located in the Joslyn field, Alberta, Canada [75]. The data was recorded during the time interval from October 1 to December 31, 2004, when the SAGD well was producing. The reservoir was initially cold, except for the toe region. Over time, the reservoir gradually heated up from the toe until December 31 when two-thirds of the bottom was hot.

4) Pipeline leak detection: Minor leaks in pipelines can develop into major disasters. Detecting pipeline leaks by pressure drop or mass balance is difficult and imprecise in locating the leak [77]. The Raman-based fiber-optic DTS can capture changes in pipeline surface temperature, which means that DTS can instantly detect leaks and even make it easier to find the leak's location [78]. An optical fiber cable can be attached directly to the surface of the pipeline or it can be buried next to the pipeline. In some cases, optical fiber cables, used for long-distance communications, are laid along with pipelines. Because telecom fiber-optic cable usually contains unused spare fibers (dark fibers), the dark fibers can be used to detect leaks along pipelines with minimal additional cost. In case a pipeline is transporting a liquid/gas, the optical fiber cable is laid below/above the pipeline surface (Fig. 16). Figs. 16(a) and 16(b) show representative examples of oil and gas leak through pipelines, which respectively raise and lower the temperature of the optical fiber cable at the site of the leak.

There are some concerns raised regarding damaging the pipeline's coating used to prevent corrosion and health-andsafety limitations on personnel working near pipelines. Thus, when possible such as in the case of a buried pipeline, it is recommended to avoid attaching the fiber-optic cable directly to the pipeline's surface and instead lay the fiber below/above a pipeline carrying liquid/gas. For example, the product literature for DTS systems indicates that the fiber can be laid down $10.2 \mathrm{~cm}$ to $15.2 \mathrm{~cm}$ below a pipeline carrying a liquid [78]. The near-field temperature of the surrounding soil is impacted by buried pipeline with internal fluid flow. The 
possibility of detecting small leaks depends not only on the specification of the DTS system, but also on the characteristics of the soil. In the event of small leaks, for example, there is a strong possibility for petroleum products released from a pipeline through a high-water-content soil to move as discrete fingers. This is considered the worst-case scenario for leak detection using DTS system because the leaked product may not contact the fiber directly. Thus, the leak detectability of a DTS system requires on-site calibration. Besides, to reduce the false alarm rate of leak detection, it is important to calibrate the DTS system so that the background soil temperature does not fluctuate significantly during the DTS resolve time.
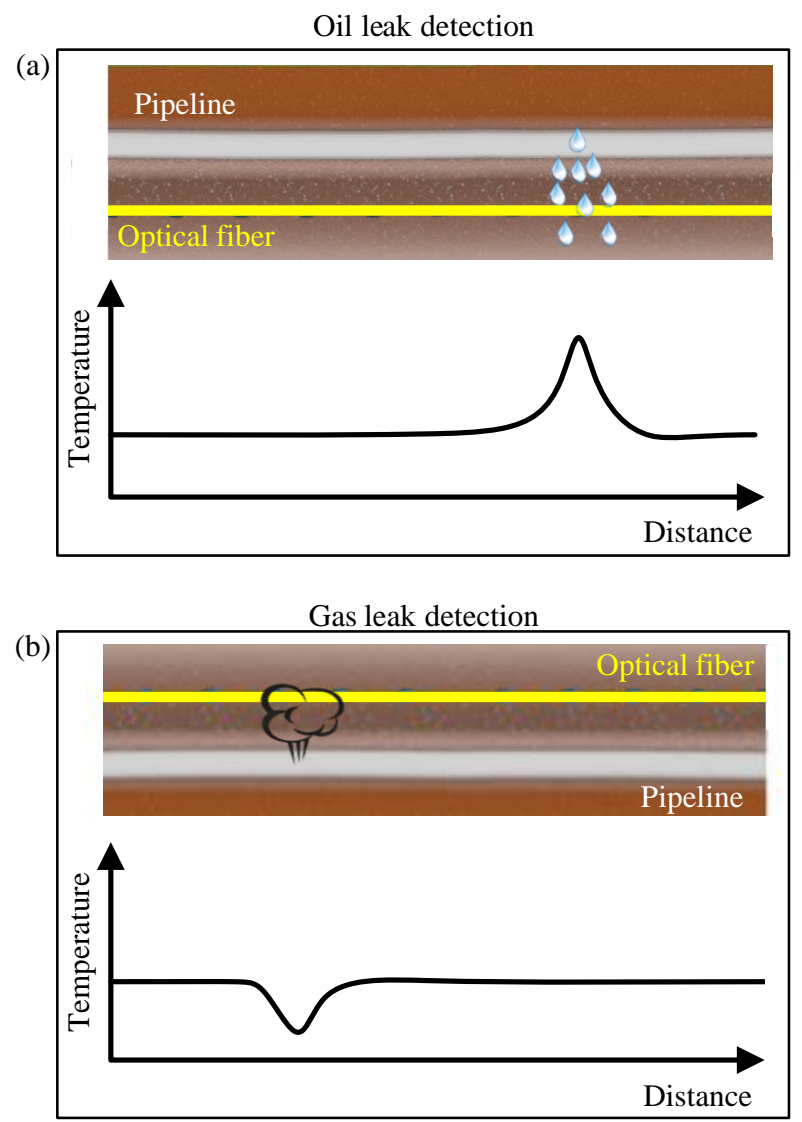

Fig. 16. Pipeline leak detection using a fiber-optic DTS when the pipeline is transporting oil (a) or gas (b).

\section{HYBRID DAS-DTS SYSTEM USING A MMF}

As mentioned earlier, fiber-optic DTS and DAS have become vital fragments in the oil and gas industry. For simultaneous distributed temperature and acoustic sensing in a well, MMF and SMF are deployed downhole in a capillary tube, along with the use of two individual DTS and DAS interrogation units on the surface [79]. The DTS and DAS system typically use the MMF and SMF, respectively. This is because the DTS relies on recording the Stokes and AntiStokes Raman signal that are of relatively weak powers, as aforementioned in Section III-A. Consequently, high power is required to be launched into the optical fiber to obtain a reasonable SNR for the DTS system. Hence, MMF is the preferred platform for the Raman-based DTS as the standard MMF has a large effective area and a high threshold power of nonlinearity that can support the needed high injected pump power, without degrading the performance of the DTS system [80]. In contrast, since the DAS operation depends on the coherent interference of Rayleigh signals that are reflected by scattering centers along the fiber, the MMF typically produces significant noises in DAS systems. This is because the standard MMF supports propagating a large number of modes and each mode has its own interference signature; consequently, the modes produce a resultant Rayleigh signal almost independent of vibration. As a result, MMF and SMF are ideal for DTS and DAS, respectively [80].

Having two separate temperature and vibration sensors increases the overall cost of well and pipeline monitoring. Another major challenge is that a myriad of producing oil and gas wells comprise only MMFs. This is because the fiber-optic DTS was explored many years before the DAS counterpart. Installing a new SMF in a production well is impractical, which prevents distributed vibration sensing within the well. As a result, for the oil and gas industry, there are strong economic and technical motivations to utilize the widespread MMF for distributed hybrid acoustic-temperature sensing.

Here, we develop a MMF-based hybrid DAS-DTS system that is tested in a field experiment. Vibration locations and frequencies, and temperature profile are simultaneously measured on-site along a standard MMF deployed in an oil well. We published the detailed design of the hybrid sensing system and its calibration process in a laboratory environment in [80], [81].

The operation principle of our design is shown schematically in Fig. 17, where we simultaneously satisfy the operational requirements of the DAS and DTS system. The optical pulses are initially launched throughout a SMF-based circulator. The circulator second port's SMF transfers the fundamental mode to a MMF, via the center-launching technique [82], [83], such that the MMF operates in a quasisingle-mode (QSM) state. In the backward direction, the AntiStokes and Stokes Raman signals are filtered out using a WDM MUX/DEMUX for temperature sensing. In contrast, the Rayleigh backscattered signal in this design is dominant by the fundamental mode because of the MMF's QSM operation [84]. The SMF of the circulator's second port acts as a spatial filter, in the backward direction, to collect only the fundamental mode of the Rayleigh signal and discards its other higher-order modes. The spatially filtered Rayleigh signal can be utilized for vibration sensing, with significant mitigation of the noise produced by the large number of modes supported by the MMF.

Fig. 18 shows the experimental setup of our reported MMFbased hybrid DAS-DTS system. An ultra-narrow linewidth laser produces a CW light of a $1550-\mathrm{nm}$ wavelength, a 40 $\mathrm{mW}$ power, and a $100-\mathrm{Hz}$ linewidth. An AOM converts the $\mathrm{CW}$ light into optical pulses of a $10-\mathrm{kHz}$ repetition rate and a $100-n s$ width. In order to satisfy the operation requirement of the DTS system, an EDFA is then used to amplify the power of the optical pulses. Next, the amplified optical pulses are routed throughout a SMF-based circulator towards the center- 


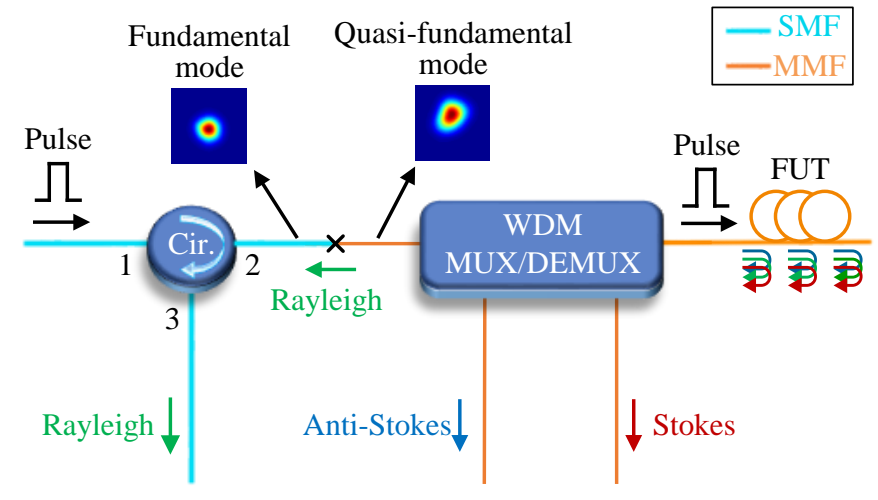

Fig. 17. Operation principles of the MMF-based hybrid DAS-DTS. Cir., circulator.

launching splicing point that mainly excites the fundamental mode in a standard 50/125 $\mu \mathrm{m}$ MMF deployed in this system. In the backward direction, the Rayleigh, Anti-Stokes Raman, and Stokes Raman signals are spectrally separated using the WDM MUX/DEMUX. The Rayleigh signal is amplified using another EDFA which amplified spontaneous emission noise is suppressed using a fiber Bragg grating (FBG), and the filtered Rayleigh signal is then detected using a PD. On the other hand, the Anti-Stokes and Stokes Raman signals are separately detected via two APDs. The analog signals from the PD/APDs are digitized using a DAQ card that includes an analog-todigital converter (ADC) and a field-programmable gate array (FPGA). The Rayleigh digital signal is continuously saved in a solid-state drive (SSD), using the FPGA's data streaming module. Meanwhile, the FPGA determines the block averaging for the Anti-Stokes and Stokes Raman signals, to improve the SNR of the DTS system. Since the electrical pulses that drive the AOM are generated by a pulse generation module controlled via the FPGA, the Rayleigh data streaming and Raman block averaging can be perfectly synchronized.

Next, we build a prototype of the hybrid sensing system for field testing. The prototype is used to simultaneously monitor vibration and temperature along a MMF extended within a wellbore. The MMF is standard of a 50/125- $\mu \mathrm{m}$ core/cladding diameter and a $\sim 3.8-\mathrm{km}$ length. Fig. 19(a) shows the developed prototype on-site while it is recording and processing the vibration and temperature data. Our team also developed a software that simultaneously visualizes the vibrations amplitudes and frequencies and temperatures along the MMF, in the field without the need for offline signal processing. This is significant for decision-makers to rapidly take critical actions, especially those related to safety. To the best of our knowledge, this is the first field testing of a MMFbased hybrid DAS-DTS system.

Fig. 19(b) provides the vibration information along the FUT, over a 2-s period, which corresponds to processing 20,000 consecutive Rayleigh traces. The results of Fig. 19(b) demonstrate that the oil well has a dynamic environment along its depth. Applying the fast Fourier transform (FFT) to the vibrations located near the wellhead, as marked in Fig. 19(b), Fig. 19(c) shows the power spectrum of these vibrations. The obtained vibration results could be fed to various application models; however, explaining what is happening within the well environment is beyond the scope of this work. The temperature profile along the MMF can be simultaneously measured (Fig. 19(d)). The system measures $\sim 30.7{ }^{\circ} \mathrm{C}$ and $\sim 93.5^{\circ} \mathrm{C}$ average temperatures on the surface and wellhead, respectively. The temperature gradually increases downhole such that it reaches $\sim 100.4{ }^{\circ} \mathrm{C}$ at the bottom of the oil well. This work would find significant interest not only for the downhole monitoring but also for other applications and industries.

\section{FIBER-OPTIC DISTRIBUTED TEMPERATURE AND STRAIN SENSING (DTSS)}

based on Brillouin Scattering In general, Brillouin-based distributed fiber-optic sensors suffer from cross-sensitivity to temperature and strain [85], making them less commonly used in the oil and gas industry. In case an environment has simultaneous dynamic changes in temperature and strain, some techniques can be used in a Brillouin-based distributed fiber-optic sensor to distinguish between temperature and strain [86]-[95]. With proper temperature and strain discrimination, Brillouin scattering can be exploited to simultaneously sense temperature and strain, the so-called DTSS. Otherwise, if we consider the target application of temperature and strain sensing separately, Brillouin-based sensors can also be used for DTS or distributed strain sensing (DSS) individually. In the oil and gas industry, Brillouin-based fiber-optic DTSS is primarily used for pipeline monitoring [31], [32], [96]-[105] with a few other well integrity-related applications [106].

The Brillouin-based fiber-optic DTSS market for the oil and gas industry was valued at USD 56 million in 2020 and is expected to reach USD 74.7 million by 2026 , at a CAGR of $4.8 \%$ between 2021 and 2026 [35]. Unlike the fiber-optic DAS and DTS markets, which are expected to be led by the oil and gas industry, the infrastructure industry is anticipated to lead the fiber-optic DSS market between 2021-2026.

\section{A. Operation principle of DTSS}

Brillouin-based distributed fiber-optic sensors have been rapidly developed over the past 30 years [16], after their first proposal in 1989 [107]-[109]. Fiber-optic DTSS can be achieved via one of two systems: Brillouin optical timedomain reflectometry (BOTDR) [110] and Brillouin optical time-domain analyzer (BOTDA) [111]. The operation principles of BOTDR and BOTDA are based on spontaneous Brillouin scattering (SpBS) and SBS, respectively. BOTDR has the advantage of having a simple experimental setup, with only one FUT pumping end, making it more convenient to deploy. In contrast, the BOTDA scheme is relatively complex and requires two FUT pumping ends. Nevertheless, BOTDA has a higher SNR than BOTDR, due to the essential principle of SBS with its higher power compared to SpBS [34]

A typical BOTDR experimental setup with heterodyne detection method is shown schematically in Fig. 20(a) [112]. A narrow linewidth laser generates a CW light that is divided by a coupler into two paths, a pump light and a local oscillator 


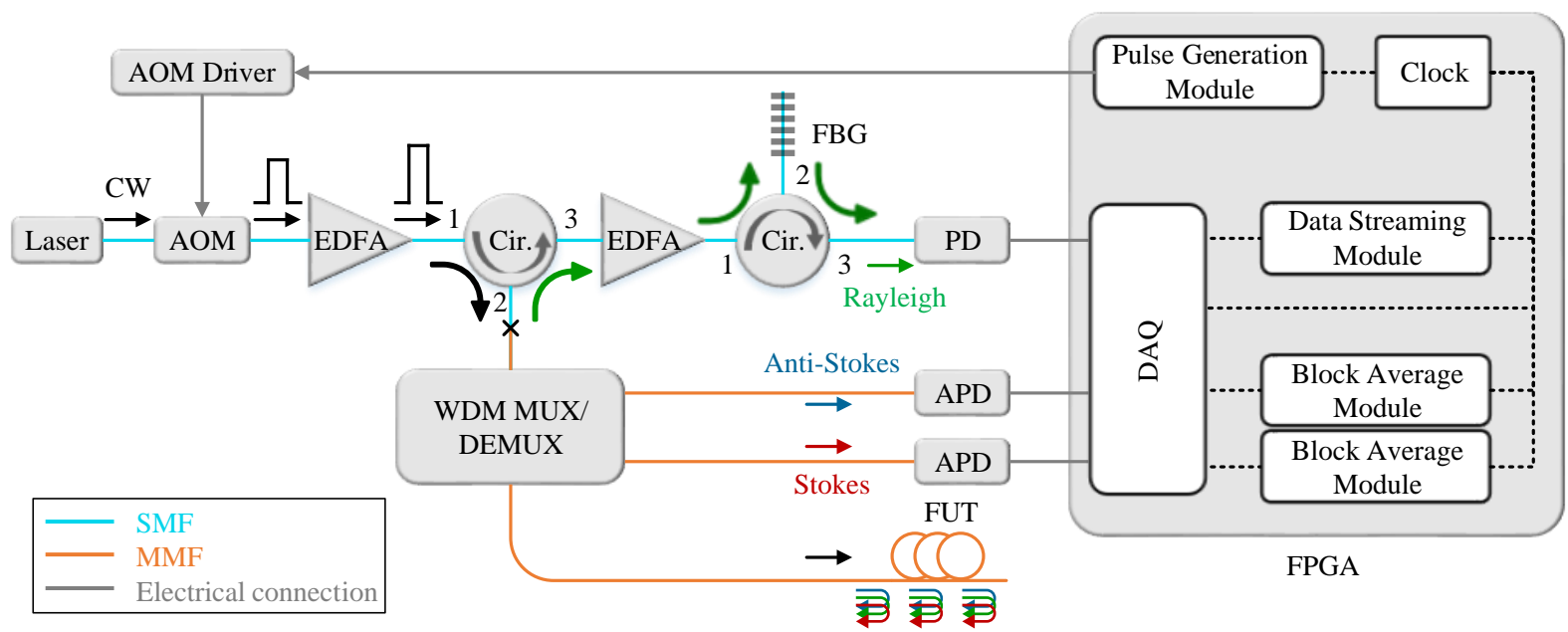

Fig. 18. Experimental setup of the MMF-based hybrid DAS-DTS. Cir., circulator.

(a)

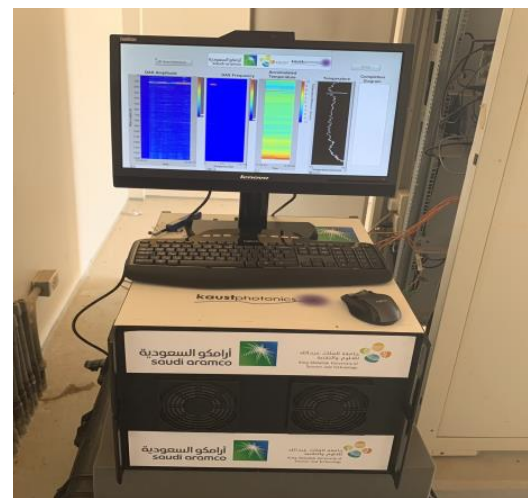

(b)
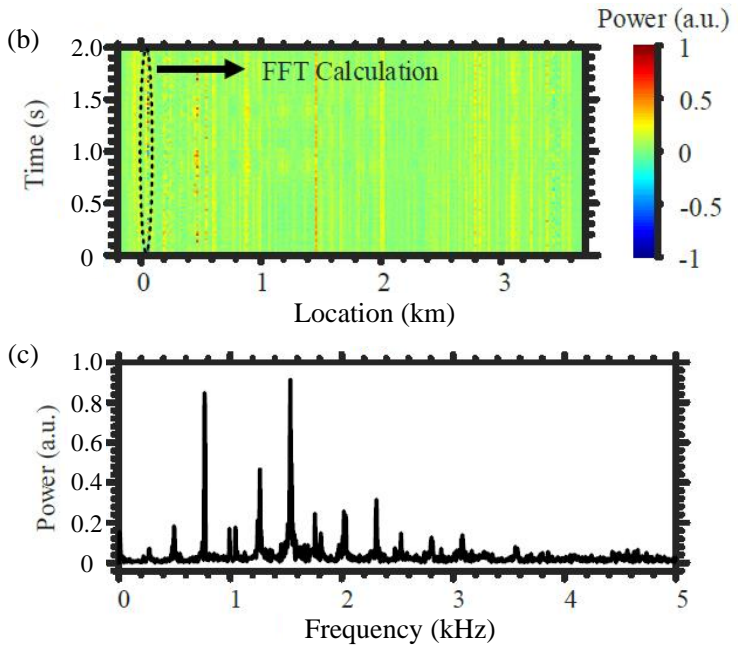

(d)

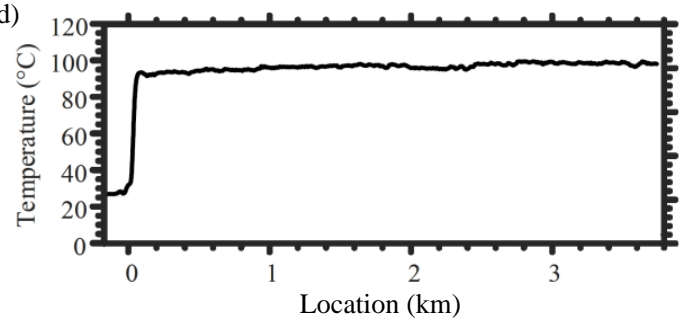

Fig. 19. (a) Prototype of the MMF-based hybrid DAS-DTS in the field. (b) Vibration information within the oil well. (c) Power spectrum of the vibrations located near the wellhead. (d) Temperature profile recorded from the surface down to the bottom of the oil well.
(LO) light. The pump light is modulated to optical pulses via an EOM driven by a function generator. The optical pulses are then amplified with an EDFA and launched into a SMF through a circulator. As the pump pulses propagate along the $\mathrm{SMF}, \mathrm{SpBS}$ signal is backscattered. The backscattered signal is directed via the circulator to be mixed with the LO light inside another coupler to generate a Brillouin beat-signal, which frequency is around $11 \mathrm{GHz}$ with the commonly used 1550 $\mathrm{nm}$ light source and SMF. The Brillouin beat-signal is then detected and recorded by a PD and a DAQ, respectively.

In contrast, the typical BOTDA system is shown in Fig. 20(b) [113], where the CW light output from a narrow linewidth laser is split into two branches by a coupler. The light at the upper branch is modulated by an EOM biased at the null-point to suppress the optical carrier and driven by a frequency controller to provide the probe light. The probe light is amplified with an EDFA and then injected into a SMF after passing through an isolator (ISO), which blocks the light in the reverse direction. At the lower branch, the CW light is modulated by another EOM to generate pump pulses. The pump pulses are also amplified with another EDFA whose ASE noise is discarded by a filter. Then, the pump pulses are injected into the SMF in the reverse direction to amplify the probe light along the SMF via the SBS effect. The amplified probe signal is then spectrally filtered to remove its highfrequency sideband, detected by a PD, and recorded with a DAQ.

In the mechanism of SpBS, only one incident pump light enters the medium and a scattered Brillouin Stokes light of a lower frequency is generated. SpBS is caused mainly by thermally generated acoustic phonons, which has relatively weak intensity [34]. On the contrary, in the SBS mechanism, two lights of different frequencies enter the medium in the reverse direction. The energy is transferred from the pump light to the lower frequency Brillouin Stokes (probe) light. SBS is caused by the electrostriction from the interference of these two lights, which significantly enhances the thermally generated sound wave [34].

Generally in Brillouin scattering, the pump light of fre- 

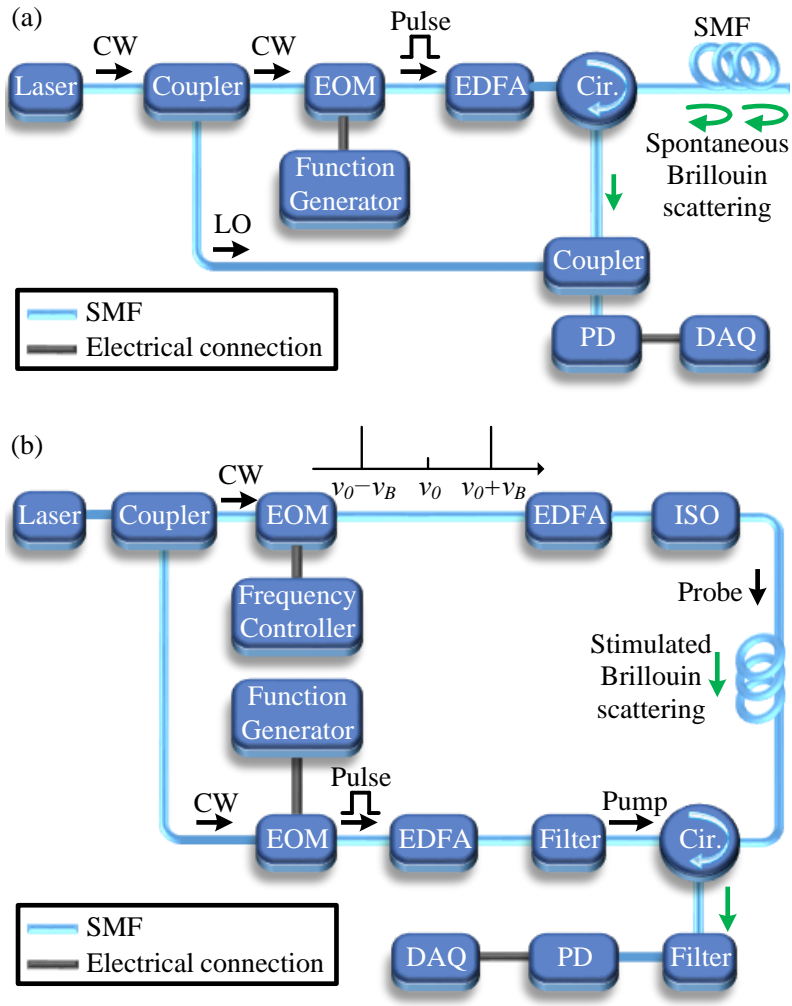

(c) Scattered wave

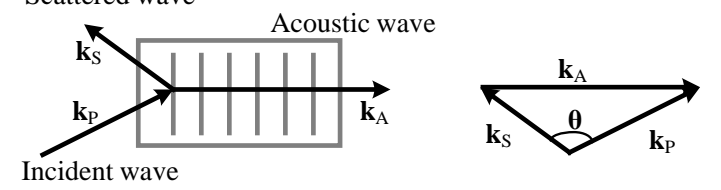

(d)

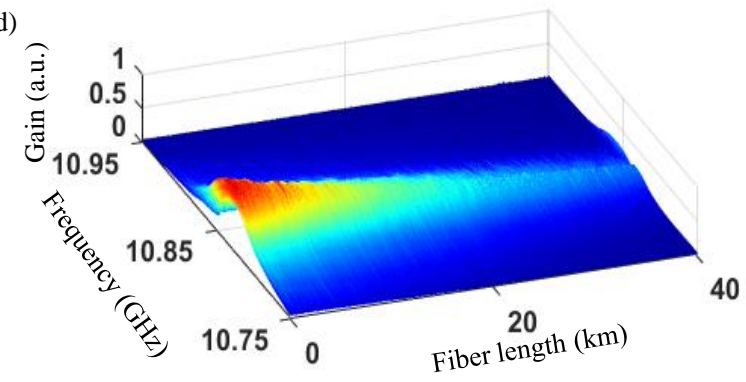

Fig. 20. Experimental setup of the BOTDR (a) and BOTDA (b). (c) Brillouin scattering in the Bragg diffraction regime. (d) Representative example of measured BGSs along a FUT.

quency $\nu_{P}$ (wave vector $\mathbf{k}_{P}$ ), the scattered light of frequency $\nu_{S}$ (wave vector $\mathbf{k}_{S}$ ), and the acoustic wave of frequency $\nu_{A}$ (wave vector $\mathbf{k}_{A}$ ) satisfy the Bragg condition for energy and momentum conservation [67]:

$$
\begin{aligned}
& \nu_{P}=\nu_{S}+\nu_{A}, \\
& \mathbf{k}_{P}=\mathbf{k}_{S}+\mathbf{k}_{A} .
\end{aligned}
$$

As shown in Fig. 20(c), the Brillouin scattering process can be described as a scattering of light in a diffraction grating moving at the velocity of sound. Generally, the Brillouin scattering can occur in all angular directions. However, only the forward and backward directions are allowed for transmission in the optical fiber. As it can be derived from Eqs. 11 and
12, the forward direction scattering vanishes while only the backscattered light "survives". The acoustic wave frequency $\nu_{A}$ represents the frequency difference between the pump light and the scattered light, also commonly called the Brillouin frequency shift (BFS) $\nu_{B}$, which can be expresses as [67]:

$$
\nu_{A}=\nu_{B}=\frac{2 n V_{A}}{\lambda_{P}},
$$

where $n$ is the effective refractive index of fiber, $V_{A}$ is the velocity of the acoustic wave, and $\lambda_{P}$ is the pump light wavelength in vacuum. Thus, the BFS depends on the intrinsic properties of the optical fiber ( $n$ and $V_{A}$ ), which are functions of strain and temperature. It has been found that the BFS in optical fibers is linearly related to the strain $(S)$ and temperature $(T)$, i.e., [85]:

$$
\nu_{B}(S, T)=C_{S} \cdot \Delta S+C_{T} \cdot \Delta T+\nu_{B}\left(S_{0}, T_{0}\right),
$$

where $\Delta \nu_{S}$ and $\Delta \nu_{T}$ denote respectively the strain and temperature change, $C_{S}\left(C_{T}\right)$ is the BFS strain (temperature) coefficient, and $\nu_{B}\left(S_{0}, T_{0}\right)$ is a reference BFS measured at predetermined strain $S_{0}$ and temperature $T_{0}$. When the acoustic wave decays exponentially, the Brillouin gain spectrum (BGS) has a Lorentzian lineshape [85]:

$$
g(\nu)=\frac{g_{B}}{1+\left[\left(\nu-\nu_{B}\right) /\left(\Delta \nu_{B} / 2\right)\right]^{2}},
$$

where $g(\nu)$ represents the Brillouin gain, $\nu$ is the frequency difference between the pump light and scattered light, $g_{B}$ is the Brillouin peak gain, and $\Delta \nu_{B}$ is the Brillouin gain bandwidth.

The local BGSs along the whole FUT can be obtained using any of the BOTDR and BOTDA systems, as shown in the representative example of Fig. 20(d). Since the BFS is the central frequency of the BGS, Lorentzian curve fitting (LCF) can be used to estimate the BFS value [114]. Thus, using Eq. 14, the strain and temperature along the FUT can be measured.

In practical applications, to distinguish between temperature and strain, techniques based on Rayleigh or Raman scattering can be combined with Brillouin-based DTSS systems for temperature and strain separation [86], [87]. In addition, a sensing fiber can be used with a specially designed strain-isolating jacket to obtain the temperature profile separately. Then, the strain information can be collected using another typical sensing fiber with compensation using the separately measured temperature [31]. Furthermore, measuring BFS together with Brillouin intensity, bandwidth, or birefringence can provide temperature and strain information separately [88]-[90]. Besides, some specialty optical fibers (SOF) that provide several BFSs can be utilized for the temperature and strain discrimination, including multi-core fiber (MCF) [91], photonic crystal fiber (PCF) [92], large-effective-area fiber (LEAF) [93], dispersion compensating fiber (DCF) [94], and few-mode fiber (FMF) [95].

It is worth highlighting that the forward Brillouin scattering (FBS), also known as the guided acoustic-wave Brillouin scattering (GAWBS) has also been investigated for sensing purposes recently [115]-[121]. The typical Brillouin-based distributed fiber-optic sensors are based on backward spontaneous/stimulated Brillouin scattering, which involves the 
interaction of the injected light with the longitudinal acoustic waves in optical fibers. In contrast, the GAWBS relies on the acoustic waves of radial or torsional-radial modes, which propagate in the radial and circumferential directions instead of the longitudinal direction in fibers [120], [121]. The scattered spectrum of GAWBS contains dozens of peaks at frequencies below $1 \mathrm{GHz}$, which is also different from that of the backward Brillouin scattering. It is difficult for the sensors based on backward Brillouin scattering to detect ambient measurands that do not directly perturb the fiber core. For example, the BFS of the backward Brillouin scattering is not affected by ambient acoustic impedance. However, the surrounding medium affects the boundary conditions of the acoustic waves at the outer boundary of the fiber cladding, and thus affects the GAWBS. As a result, using a standard SMF, GAWBS can be used to measure the acoustic impedance of the ambient medium along with temperature and strain. Then the acoustic impedance can be used to analyze chemical species, e.g., the type and concentration of solutions [115], [116], which is a unique advantage of GAWBS. GAWBS-based sensors may be deployed for underground oil layer detection and other potential applications in the oil and gas industry in the future. However, it still needs some development to become reliable, due to the challenges of achieving longdistance distributed sensing and the requirement of having a bare fiber to provide direct contact with the surrounding medium.

\section{B. Applications of DTSS in the oil and gas industry}

In the oil and gas industry, Brillouin-based fiber-optic DSS can be used to monitor pipelines [31], [32], [96]-[105] and well integrity [106]. Here, we present some representative petroleum-related applications of the DTSS.

1) Pipeline leak and: deformation monitoring Long-time operation of pipelines in a harsh field environment may corrode and even damage pipelines. In addition, pipelines are also exposed to ambient environmental hazards such as landslides, earthquakes, and human activities. Thus, distributed deformation and leak monitoring of pipelines achieved by measuring temperature and/or strain using fiber-optic DTSS are highly demanded to detect these hazard conditions and prevent damage in advance with some interventions.

Fig. 21(a) shows an example of using a fiber-optic DTSS system for deformation monitoring of a buried 35-year-old gas pipeline in Italy [31]. Three fiber-optic strain sensing cables and one temperature sensing cable are installed along the entire pipeline section in parallel. The strain sensing cable is designed to make the optical fiber bonded to the pipeline without sliding, while the temperature sensing cable is designed to be isolated from external strain changes with a loose tube. The three strain sensing cables are installed at $0^{\circ}$, $120^{\circ}$, and $-120^{\circ}$ with respect to the pipeline axis, respectively, while the temperature sensing cable is installed at $0^{\circ}$ position on the pipeline to compensate for temperature for the strain measurement. Then the pipeline is loaded with soil and thereby deformed. Fig. 21(b) shows some successful measurements of the averaged strain distribution along the pipeline as measured by the three strain sensing cables (marked as SMARTapes 1, 2, and 3 in Fig. 21(b)), after compensation for the temperature measured by the temperature sensing cable. Using the strain distribution data with this installation technique, the curvature and deformed shape of the pipeline can also be estimated [31], [97]. Additional fiber-optic sensing cables can also be installed around the pipeline to provide more detailed analyses regarding the environmental conditions, and to investigate the relationship between the ambient conditions and the resulting deformation of the pipeline.

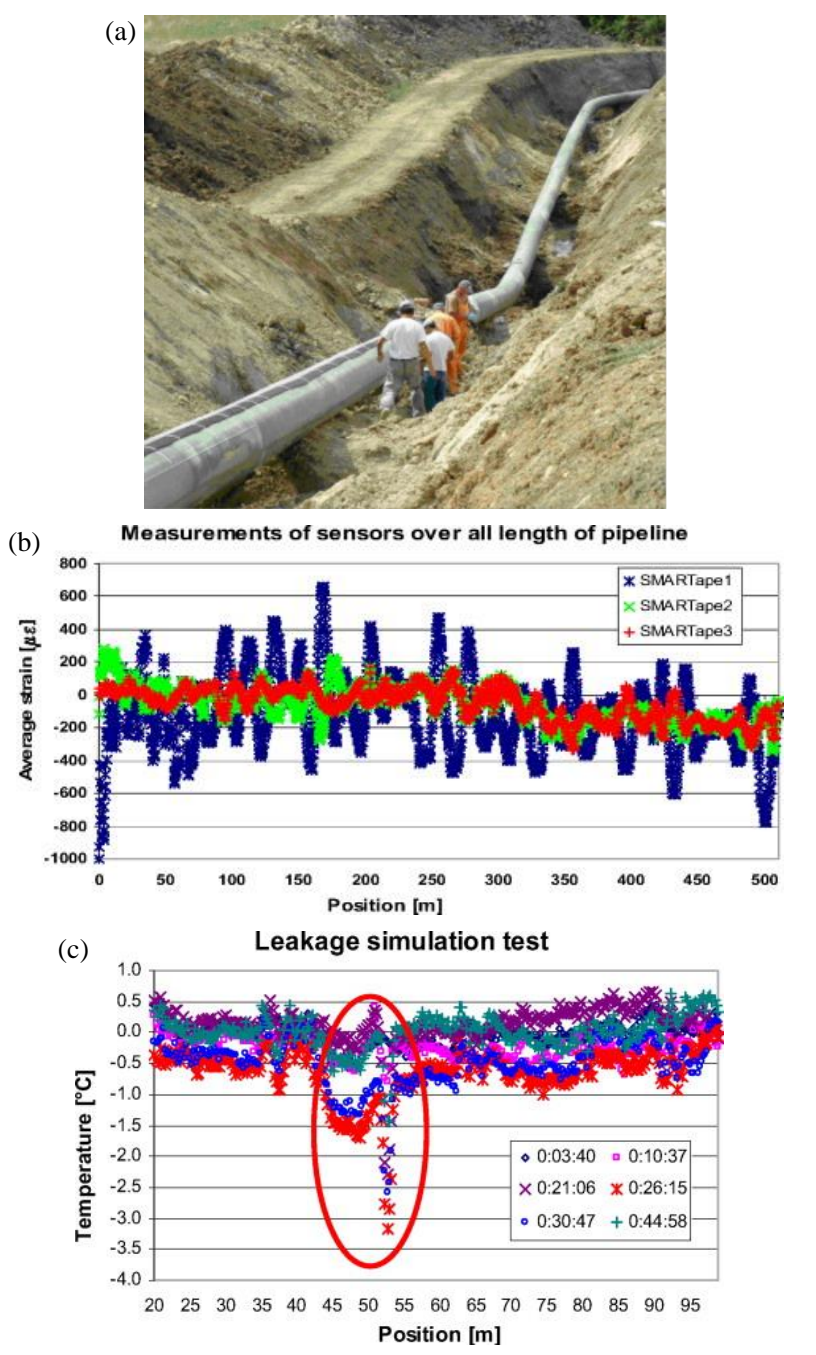

Fig. 21. (a) Installing the DTSS cables on the gas pipeline. (b) Strain distribution along the monitored part of the pipeline. (c) Temperature distribution results of leak test, where leak is detected using the temperature change. (Reproduced from [31] with permission).

Another gas detection experiment was successfully performed using the DTSS system. Carbon dioxide was released at a single specific position on the upper surface of the pipeline to simulate a gas leak. Then, the temperature profile along the pipeline was collected using the temperature sensor of the DTSS, which together with the reference temperature information obtained before the carbon dioxide was released are shown in Fig. 21(c). The results show that the leak can be clearly observed and located. This representative example 
demonstrates the capability of the Brillouin-based DTSS to implement practical pipeline leak and deformation monitoring.

Regarding the deployment of fiber-optic cables for pipeline deformation monitoring using DSS, the optimal choice is to attach the optical cable directly to the pipeline surface for maximum strain detection sensitivity. However, bearing in mind that in some cases this may affect the protective coating of the pipeline or be dangerous for operators to attach fibers directly to the pipeline during pipeline construction, it is preferable to lay the optical cable a few inches above the pipeline during the backfill. This is also convenient for possible splicing and maintenance in the future. In this case, although the direct strain information of the pipeline cannot be obtained, the measured ambient strain information is still very important and useful for detecting hazardous conditions around the pipeline.

As noted, both Raman and Brillouin scattering can be exploited for DTS measurement. The sensing range of the Raman-based DTS is usually limited to tens of kilometers, which is relatively short compared with the ultra-long sensing range (beyond $150 \mathrm{~km}$ ) of the Brillouin-based distributed sensors for DTS/DSS/DTSS. The limited sensing range of the Raman-based DTS is due to the weak intensity of the spontaneous Raman light, which requires the use of high pump power in a MMF to avoid the undesired nonlinear effects and improve the SNR. However, intermodal dispersion of the MMF further limit the sensing range. In this regard, the backscattering intensity of the Brillouin-based DTSS can be highly amplified using stimulated Brillouin scattering and further the Brillouin-based DTSS utilizes SMF, which both result in having an ultra-long sensing range.

2) Well integrity: Reservoir compaction may induce buckling, tension, and shear failures in wells [106]. Fiber-optic DSS can be used to monitor well deformation in real-time, which may prevent or mitigate damage from well failures.

Axial tensile deformation is one of the critical well deformation mechanisms, which could cause cement failure and lead to loss of zonal isolation. Fig. 22(a) shows an example of using a fiber-optic DSS to detect the axial deformation of a $3.1 \mathrm{~m}$ well model, located in a laboratory environment [106]. The well model consists of an inner pipe, a cement sheath, and an outer pipe, which respectively represent the casing, cement sheath, and formation in the field. The fiber-optic cables are installed in the annular cement of the model, while a tensile load is applied on the outer pipe to produce the tensile deformation. The cables employed from Hole \#1 to Hole \#6 are used for BOTDR-based DSS, while the other two cables in Hole \#7 and Hole \#8 include FBGs for validation and comparison. It should be noted that there are several optical fiber cables between Hole \#1 and Hole \#8, some of which are specially designed temperature fiber cables. The fiber core in these cables is encased in an air- or gel-filled tube to be isolated from external strain changes to compensate for temperature change during the strain measurements with the BOTDR or FBGs. It should be noted that the exploited BOTDR sensor is a DTSS system, although we call it DSS here because it focuses on strain sensing for the well deformation detection only.

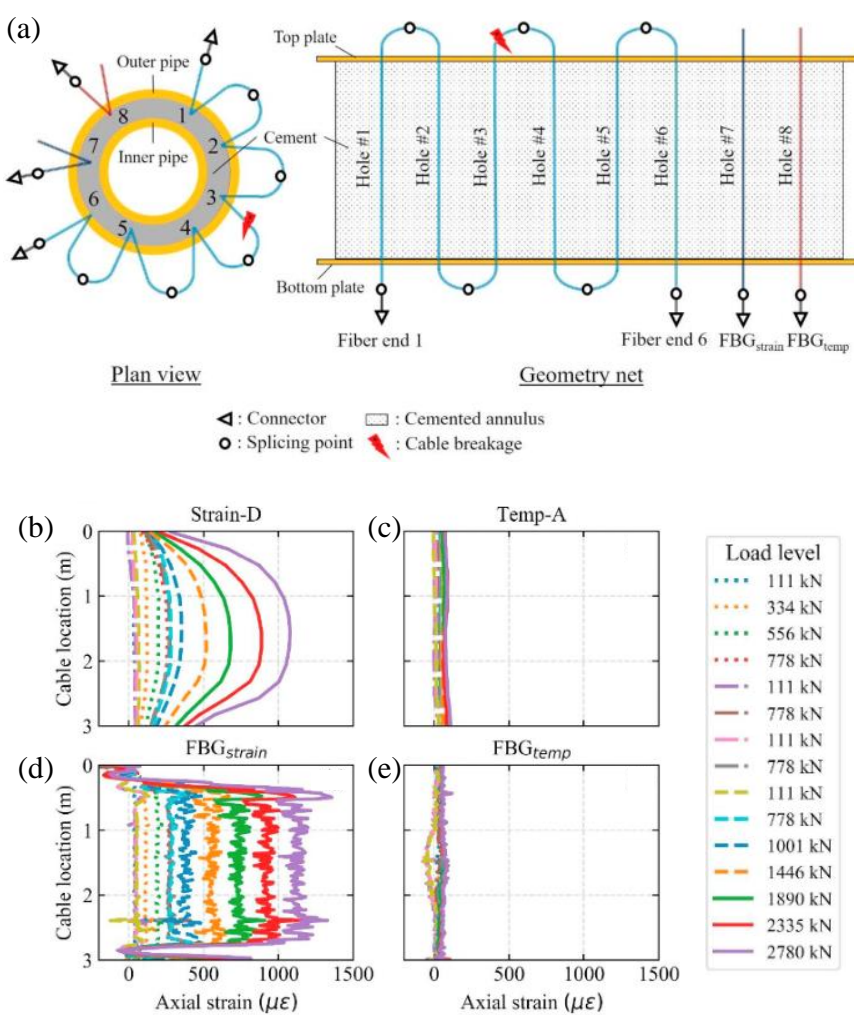

Fig. 22. (a) The configuration of the fiber-optic cables installed in the specimen for the well deformation detection. BOTDR measurements of strain (b) and temperature (c) using fiber-optic cables named Strain-D and Temp-A, respectively. FBGs measurements of strain (d) and temperature (e) using fiber-optic cables named $\mathrm{FBG}_{\text {strain }}$ and $\mathrm{FBG}_{\text {temp }}$, respectively. (Reproduced from [106] with permission).

Examples of the BOTDR-based measurements for the strain and temperature are shown in Figs. 22(b) and 22(c), respectively. For the purpose of comparison, Figs. 22(d) and 22(e) respectively show the strain and temperature measurements using the FBGs. The strain measurements of the BOTDR and FBGs are generally consistent; however, the BOTDR measurement is much smoother. This is because the strain measurement of the BOTDR is averaged within a 1-m spatial resolution of the distributed sensor. In contrast, the FBGbased sensors provide strain information at each FBG location with an interval of $2 \mathrm{~cm}$. These results highlight the key advantage of distributed fiber-optic sensing that it can monitor long distances at lower cost and less complexity, compared to single-point and quasi-distributed fiber-optic sensors. Although this work has been successful in monitoring the axial well deformation within a laboratory environment, more work is needed to apply it in the field, especially for the method of installing the fiber-optic cables.

\section{MARKET, CHALLENGES, AND FUtURE DIRECTIONS}

\section{A. Market}

In terms of market analysis, the oil and gas industry is leading the distributed fiber-optic sensing market (Fig. 23). DTS and DAS systems are the most widely used sensors in the oil and gas vertical, with significant market penetration for DAS due to its various emerging applications [35]. The 
distributed fiber-optic sensing market for the oil and gas vertical was valued at USD 517.9 million in 2020 and is expected to reach USD 768.9 million in 2026, growing at a compound annual growth rate (CAGR) of $6.7 \%$ between 2021 and 2026 [35]. This market growth for distributed fiber-optic sensing in the oil and gas industry is driven by the rising need for productivity, efficiency, and safety of operations, which can be improved with the use of distributed fiber-optic sensing. Table I summarizes a wide range of companies that sell and/or provide service for distributed fiber-optic sensors and fiberoptic cables deployed in the oil and gas industry and other related applications [16], [35].

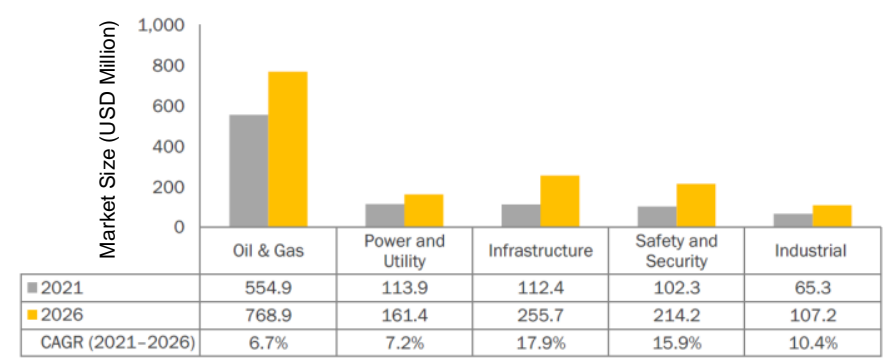

Fig. 23. Distributed fiber-optic sensing market for different industries. (Reproduced from [35] with permission).

\section{B. Challenges}

There are some major obstacles hindering growth in the deployment of distributed fiber-optic sensors. The high-cost of fiber-optic sensors poses a major challenge to the development of this market [35]. Depending on the applications, operating conditions, and the type of fiber-optic cable used, the cost of a distributed fiber-optic sensing system can be very high and still not affordable by every company that requires realtime monitoring and sensing. In addition, the installation and maintenance of these systems are also costly which makes their implementation difficult. However, as distributed fiberoptic sensing adoption becomes more rapid, improvements in manufacturing and volume pricing will result in lower costs. Additionally, lifetime continuous monitoring with fiber-optic sensors can offset the high initial cost.

Other technical challenges include the fiber darkening downhole [76], where hydrogen penetrates the metal tube around the fiber and fiber jackets to enter the fiber material causing a significant optical attenuation. However, many efforts have been reported to tackle the hydrogen darkening by using hermetic coating, tailoring the glass properties, and selecting proper operation wavelengths [122]. Such optical fiber cables with special coating and properties are now readily available for long-term downhole monitoring.

Another hurdle relates to data size as distributed fiber-optic sensors can provide continuous monitoring to the downhole environment and pipelines, producing a massive amount of data. Although continuous monitoring offers many advantages, it also comes with its own set of challenges in terms of data management, storage, visualization, and security. Cloudbased data management has recently been introduced to the oil and gas industry as an efficient mean to store, transmit, and visualize data produced by distributed fiber-optic sensors [123].

\section{Future directions}

The research community for distributed fiber-optic sensing continues to develop new technologies in this field, which should provide further application opportunities in the oil and gas industry. One of the main goals of the current research work is to improve the overall performance of distributed fiber-optic sensing. For example, introducing new modulation schemes, novel coding formats, and advanced signal processing methods may help reduce the limitations of spatial resolution, sensing range, and measuring accuracy of distributed fiber-optic sensors [16], [85], [124]. Another research direction is to explore the capabilities of fiber-optic sensors, including the distributed ones, to measure more parameters with high reliability in the harsh environment of the oil and gas industry. In laboratory environment, fiber-optic sensors have demonstrated the ability to measure various physical and chemical parameters, such as temperature, strain, vibration, pressure, curvature, rotation, electric/magnetic field, flow, liquid level, and liquid concentration [16], [125]. However, few of these technologies have been reliably adopted in the oil and gas markets. Thus, it is highly desirable to market more fiber-optic sensors, especially chemical ones, to the oil and gas industry.

Besides, for industrial applications, stability and durability are just as important as the sensitivity of the sensors. Generally, the installation schemes of optical fibers directly affect the performance and ruggedness of the sensing systems. Thus, the installation schemes will continue to be investigated and improved in the various application scenes. Furthermore, the reliability of the system under different environments in the oil and gas industry should also be researched, especially in harsh environments with high temperature and high pressure. It should be noted that novel design and/or doping methods of specialty optical fibers adopted in sensing systems may help reduce transmission loss and increase scattered light, and also increase the tolerance of the system for harsh environments [126], [127]. This would emerge new applications of distributed fiber-optic sensors in the various sectors of the oil and gas industry.

Another development trend of distributed fiber-optic sensors in the oil and gas industry is to integrate different techniques of sensing, including DAS, DTS, and DSS, to provide a distributed multiplexed sensing (DXS) system [128]-[131]. Since the present distributed fiber-optic sensors share some identical optoelectronic components and can be employed through one single optical fiber or several optical fibers inside a single tube, it should be feasible to integrate these different techniques into a DXS system based on the need, which provides multi-parameter measurements. One advantage of the integrated DXS system is that its cost is less than the sum of individual sensing systems, while it can provide more useful information rather than simply adding up the results of several systems. This is because a comprehensive analysis 
TABLE I

DISTRIBUTED FIBER-OPTIC SENSING COMPANIES WITH APPLICATIONS IN THE OIL AND GAS INDUSTRY.

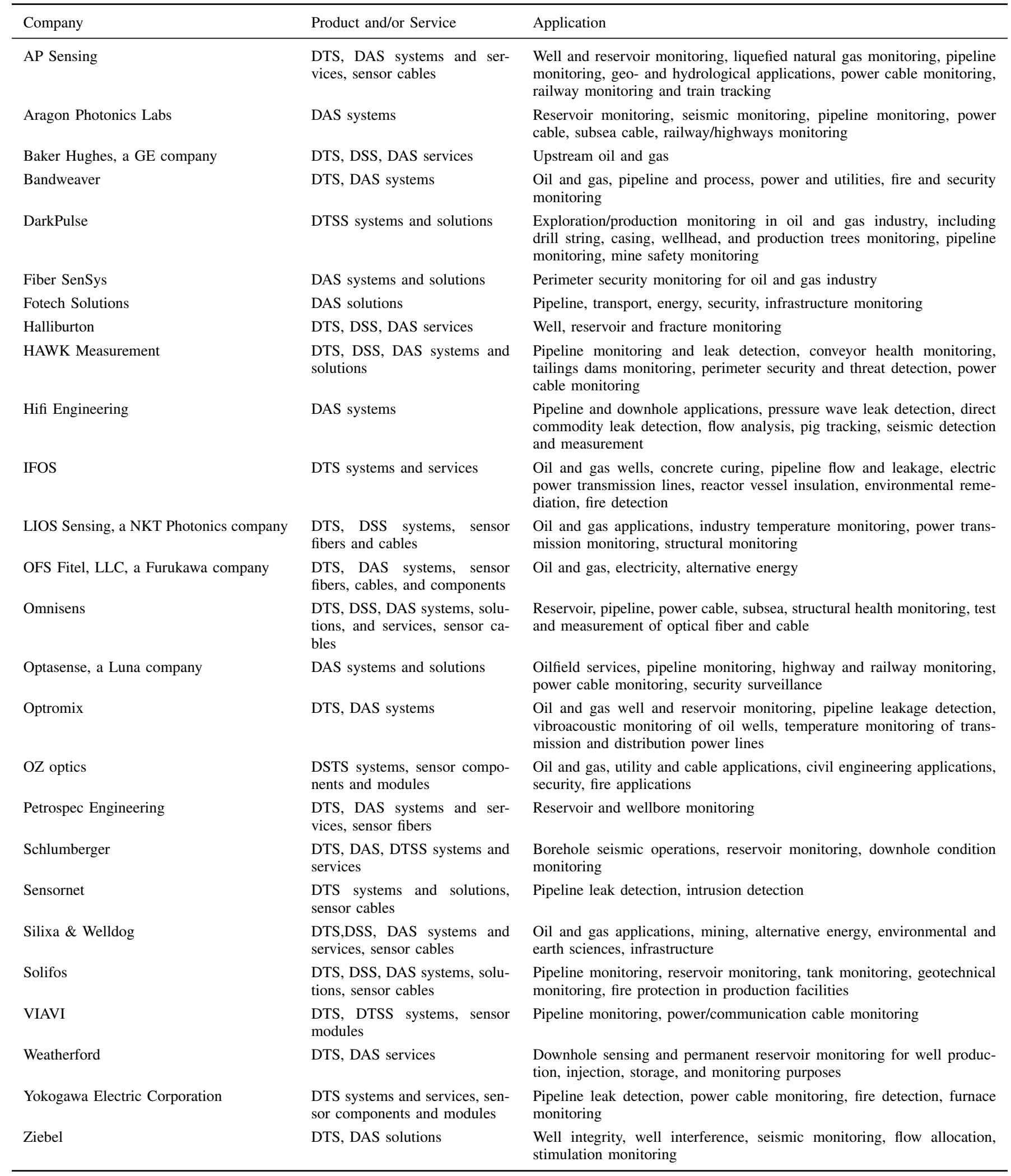


of multidimensional data at the same location would provide more profound information on the hotspot.

On the other hand, the existing challenges in the oil and gas industry will make the data-driven analyses even more important. For example, the increasing difficulty of oil recovery and public concerns of natural environment and resources will require more efficient oil recovery processes and fewer pipeline leakage events, where the data from corresponding sensing systems are necessary. The trend of integrated sensing systems and the trend of data-driven analyses will complement each other in the oil and gas industry. While the amount of data obtained and required becomes very huge, it is unrealistic to rely only on experts in this field to provide complete and detailed analyses and make decisions along with the whole process. Thus, advanced artificial intelligence (AI) and machine learning methods should be beneficial. They can help to reduce the data size and extract useful data of events, and even to make the decisions on behalf of the experts [132]. In addition, take into consideration the present and future employments of many different sensing and measuring systems in the oil and gas industry, the adoption of a cloudbased platform would be a promising solution to solve the problems of secure and reliable data transport, management, storage, analysis, visualization, and download [123], [133], [134].

\section{DISCUSSION AND CONCLUSION}

Distributed fiber-optic sensing has been used in many applications across all sectors of the oil and gas industry. The fiber-optic DAS can detect vibrations and their frequencies downhole and along pipelines. Thus, fiber-optic DAS can be used for VSP, hydraulic fracturing monitoring, downhole flow monitoring, well integrity, and pipeline intrusion detection. Raman-based DTS is another technique of distributed sensing that measures temperature along an optical fiber cable. DTS has been deployed in many petroleum applications, including downhole flow and injection monitoring, SAGD, and pipeline leak detection. We developed a prototype for a hybrid sensing system that simultaneously measures vibration and temperature along a MMF. The reported hybrid sensor relies on exciting mainly the fundamental mode into the MMF in order to satisfy the operational requirements of both the DAS and DTS. The hybrid MMF-based DAS-DTS was examined in a well to monitor the dynamic environment and temperature downhole. Various vibration events were detected along the well, and a temperature difference of $\sim 62.8^{\circ} \mathrm{C}$ was recorded between the wellhead and the surface.

We also highlighted that Brillouin-based fiber-optic DTSS can be used to monitor well integrity, and detect pipeline deformation. The growth of the fiber-optic DAS, DTS, and DSS market is mainly hampered by their high-cost, which would be mitigated by the rapid adoption of the distributed fiber-optic sensing technologies. Finally, we pointed out that improving the overall performance of the sensing systems, multi-parameter sensing, including AI and machine learning, and offering a cloud-based data management are the future research directions of distributed fiber-optic sensing.
It should be noted that there is a special class of distributed interferometer sensors, which differs from the previously discussed classical backscattering distributed fiberoptic sensors. The distributed interferometer sensors include Michelson interferometers [135], Mach-Zehnder interferometers [136]-[138], Sagnac interferometers [139], [140], and hybrid interferometers [141]-[143]. These interferometer sensors are usually used as distributed vibration sensors (DVS) and need dual interferometer structures to locate the perturbation position. Compared to the classical backscattering distributed sensors, the distributed interferometer sensors have the complexity of finding multiple intrusion sites simultaneously and have much lower locating accuracy, but can detect vibrations of higher frequencies. Although distributed interferometer sensors can find applications in pipeline monitoring [144]-[146] to detect fast dynamic intrusions, the classical backscattering distributed sensors are more widely deployed in the oil and gas industry.

\section{ACKNOWLEDGMENT}

Figure 2 was produced by Heno Hwang, a scientific illustrator at King Abdullah University of Science and Technology (KAUST).

\section{REFERENCES}

[1] BP. (2020) Energy consumption worldwide from 2000 to 2018, with a forecast until 2050. [Online]. Available: https://www.statista.com/statistics/222066/projected-globalenergy-consumption-by-source/

[2] A. Inkpen and M. H. Moffett, The global oil \& gas industry: Management, strategy and finance. PennWell Books, LLC, 2011.

[3] J. Bentham, "The scenario approach to possible futures for oil and natural gas," Energy Policy, vol. 64, pp. 87-92, 2014.

[4] T. A. Saleh and S. A. AL-Hammadi, "Insights into the fundamentals and principles of the oil and gas industry: The impact of nanotechnology," in Nanotechnology in Oil and Gas Industries. Springer, 2018, pp. $1-35$

[5] J. R. Fanchi and R. L. Christiansen, Introduction to petroleum engineering. John Wiley \& Sons, 2016.

[6] A. Hartog, B. Frignet, D. Mackie, and M. Clark, "Vertical seismic optical profiling on wireline logging cable," Geophysical Prospecting, vol. 62, no. 4-Vertical Seismic Profiling and Microseismicity Frontiers, pp. 693-701, 2014.

[7] A. Constantinou, A. Farahani, T. Cuny, and A. Hartog, "Improving das acquisition by real-time monitoring of wireline cable coupling," in 2016 SEG International Exposition and Annual Meeting. OnePetro, 2016.

[8] J. A. Veil, M. G. Puder, D. Elcock, and R. J. Redweik Jr, "A white paper describing produced water from production of crude oil, natural gas, and coal bed methane.” Argonne National Lab., IL (US), Tech. Rep., 2004.

[9] A. Y. Abukhamsin, "Inflow profiling and production optimization in smart wells using distributed acoustic and temperature measurements," Ph.D. dissertation, Stanford University, 2016.

[10] A. Bahadori, Oil and gas pipelines and piping systems: Design, construction, management, and inspection. Gulf Professional Publishing, 2016.

[11] F. Khan, R. Yarveisy, and R. Abbassi, "Risk-based pipeline integrity management: a road map for the resilient pipelines," Journal of Pipeline Science and Engineering, 2021.

[12] T. Yamate, G. Fujisawa, and T. Ikegami, "Optical sensors for the exploration of oil and gas," Journal of Lightwave Technology, vol. 35 , no. 16 , pp. $3538-3545,2017$.

[13] A. Baker, J. Gaskell, J. Jeffery, A. Thomas, T. Veneruso, and T. Unneland, "Permanent monitoring-looking at lifetime reservoir dynamics," Oilfield Review, vol. 4, no. 7, pp. 32-46, 1995. 
[14] S. Tiku, A. Veneruso, R. Etchells, and M. Pecht, "Risk factors in oil and gas well electronics compared to other electronic industries," Oil \& gas science and technology, vol. 60, no. 4, pp. 721-730, 2005.

[15] C. Baldwin, "Fiber optic sensors in the oil and gas industry: Current and future applications," in Opto-mechanical fiber optic sensors. Elsevier, 2018, pp. 211-236.

[16] P. Lu, N. Lalam, M. Badar, B. Liu, B. T. Chorpening, M. P. Buric, and P. R. Ohodnicki, "Distributed optical fiber sensing: Review and perspective," Applied Physics Reviews, vol. 6, no. 4, p. 041302, 2019.

[17] M. Amanzadeh, S. M. Aminossadati, M. S. Kizil, and A. D. Rakić, "Recent developments in fibre optic shape sensing," Measurement, vol. 128, pp. 119-137, 2018.

[18] D. A. Krohn, T. MacDougall, and A. Mendez, Fiber optic sensors: fundamentals and applications. Spie Press Bellingham, WA, 2014.

[19] J. Dakin, "Multiplexed and distributed optical fibre sensor systems," Journal of Physics E: Scientific Instruments, vol. 20, no. 8, p. 954, 1987.

[20] M. R. Kabir, A. Ali, S. Pradhan, Q. Dashti, A. Al-Jasmi, and R. Abid, "Successful implementation of real time downhole pressure temperature monitoring using fiber optic technology in deep ht-hp well in north kuwait," in IPTC 2014: International Petroleum Technology Conference. European Association of Geoscientists \& Engineers, 2014, pp. cp-395.

[21] Ö. H. Ünalmis and S. Trehan, "In-well, optical, strain-based flow measurement technology and its applications," in SPE Europec/EAGE Annual Conference. OnePetro, 2012.

[22] C. S. Baldwin, "Applications for fiber optic sensing in the upstream oil and gas industry," in Fiber Optic Sensors and Applications XII, vol. 9480. International Society for Optics and Photonics, 2015, p. 94800D.

[23] H. den Boer, J. van der Horst, R. Paleja, D. Randell, D. Joinson, P. McIvor, K. Green, R. Bartlett et al., "Flow monitoring and production profiling using das," in SPE Annual Technical Conference and Exhibition. OnePetro, 2014.

[24] G. Naldrett, C. Cerrahoglu, and V. Mahue, "Production monitoring using next-generation distributed sensing systems," Petrophysics-The SPWLA Journal of Formation Evaluation and Reservoir Description, vol. 59, no. 04, pp. 496-510, 2018.

[25] P. Stajanca, S. Chruscicki, T. Homann, S. Seifert, D. Schmidt, and A. Habib, "Detection of leak-induced pipeline vibrations using fiber-optic distributed acoustic sensing," Sensors, vol. 18, no. 9, p. $2841,2018$.

[26] X. Bao and L. Chen, "Recent progress in distributed fiber optic sensors," sensors, vol. 12, no. 7, pp. 8601-8639, 2012.

[27] G. Naldrett, T. Parker, S. Shatalin, M. Mondanos, and M. Farhadiroushan, "High-resolution carina distributed acoustic fibreoptic sensor for permanent reservoir monitoring and extending the reach into subsea fields," First Break, vol. 38, no. 2, pp. 71-76, 2020.

[28] M. Ichikawa, S. Uchida, M. Katou, I. Kurosawa, K. Tamura, A. Kato, Y. Ito, M. de Groot, and S. Hara, "Case study of hydraulic fracture monitoring using multiwell integrated analysis based on low-frequency das data," The Leading Edge, vol. 39, no. 11, pp. 794-800, 2020.

[29] K. Boone, A. Ridge, R. Crickmore, and D. Onen, "Detecting leaks in abandoned gas wells with fibre-optic distributed acoustic sensing," in IPTC 2014: International petroleum technology conference. European Association of Geoscientists \& Engineers, 2014, pp. cp-395.

[30] Z. Peng, J. Jian, H. Wen, A. Gribok, M. Wang, H. Liu, S. Huang, Z.-H. Mao, and K. P. Chen, "Distributed fiber sensor and machine learning data analytics for pipeline protection against extrinsic intrusions and intrinsic corrosions," Optics Express, vol. 28, no. 19, pp. 27277 $27292,2020$.

[31] D. Inaudi and B. Glisic, "Long-range pipeline monitoring by distributed fiber optic sensing," Journal of pressure vessel technology, vol. 132, no. $1,2010$.

[32] S. Zhang, B. Liu, and J. He, "Pipeline deformation monitoring using distributed fiber optical sensor," Measurement, vol. 133, pp. 208-213, 2019.

[33] A. Rogers, "Distributed optical-fibre sensing," Measurement Science and Technology, vol. 10, no. 8, p. R75, 1999.

[34] R. W. Boyd, Nonlinear optics. Academic press, 2020.

[35] MarketsandMarkets, "Distributed fiber optic sensor market with covid19 impact analysis-global forecast to 2026," 2021.

[36] J. Dakin and C. Lamb, "Distributed fibre optic sensor system gb2222247a," 1990.

[37] H. F. Taylor and C. E. Lee, "Apparatus and method for fiber optic intrusion sensing," Mar. 16 1993, uS Patent 5,194,847.
[38] R. Juškaitis, A. Mamedov, V. Potapov, and S. Shatalin, "Distributed interferometric fiber sensor system," Optics letters, vol. 17, no. 22, pp. $1623-1625,1992$.

[39] — , "Interferometry with rayleigh backscattering in a single-mode optical fiber," Optics letters, vol. 19, no. 3, pp. 225-227, 1994.

[40] I. Ashry, Y. Mao, M. S. Alias, T. K. Ng, F. Hveding, M. Arsalan, and B. S. Ooi, "Normalized differential method for improving the signalto-noise ratio of a distributed acoustic sensor," Applied optics, vol. 58, no. 18 , pp. 4933-4938, 2019.

[41] W. Seo, "Fiber optic intrusion sensor investigation," Ph.D. dissertation, Texas A\&M University, 1994.

[42] M. Ren, "Distributed optical fiber vibration sensor based on phasesensitive optical time domain reflectometry," Ph.D. dissertation, Université d'Ottawa/University of Ottawa, 2016.

[43] X. Bao, D.-P. Zhou, C. Baker, and L. Chen, "Recent development in the distributed fiber optic acoustic and ultrasonic detection," Journal of Lightwave Technology, vol. 35, no. 16, pp. 3256-3267, 2016.

[44] R. Posey, G. Johnson, and S. Vohra, "Strain sensing based on coherent rayleigh scattering in an optical fibre," Electronics Letters, vol. 36 , no. 20, pp. 1688-1689, 2000.

[45] Z. Pan, K. Liang, Q. Ye, H. Cai, R. Qu, and Z. Fang, "Phasesensitive otdr system based on digital coherent detection," in Asia Communications and Photonics Conference and Exhibition. Optical Society of America, 2011, p. 83110S.

[46] A. Hartog and K. Kader, "Distributed fiber optic sensor system with improved linearity," Oct. 27 2015, uS Patent 9,170,149.

[47] J. Pastor-Graells, H. F. Martins, A. Garcia-Ruiz, S. Martin-Lopez, and M. Gonzalez-Herraez, "Single-shot distributed temperature and strain tracking using direct detection phase-sensitive otdr with chirped pulses," Optics express, vol. 24, no. 12, pp. 13 121-13 133, 2016.

[48] A. Masoudi, M. Belal, and T. Newson, "A distributed optical fibre dynamic strain sensor based on phase-otdr," Measurement Science and Technology, vol. 24, no. 8, p. 085204, 2013.

[49] A. Masoudi and T. P. Newson, "High spatial resolution distributed optical fiber dynamic strain sensor with enhanced frequency and strain resolution," Optics letters, vol. 42, no. 2, pp. 290-293, 2017.

[50] J. Toulouse, "Optical nonlinearities in fibers: review, recent examples, and systems applications," Journal of lightwave technology, vol. 23, no. 11, p. 3625, 2005.

[51] X. Hu, C. Cao, Z. Meng, W. Chen, Y. Lu, J. Wang, and M. Chen, "The modulation instability induced temporal fluctuation of the optical pulses in optical fiber sensing systems," in Optical Fiber Sensors. Optical Society of America, 2018, p. WF50.

[52] O. H. Waagaard, E. Rønnekleiv, A. Haukanes, F. Stabo-Eeg, D. Thingb $\varnothing$, S. Forbord, S. E. Aasen, and J. K. Brenne, "Real-time low noise distributed acoustic sensing in $171 \mathrm{~km}$ low loss fiber," OSA Continuum, vol. 4, no. 2, pp. 688-701, 2021.

[53] T. Alsos, "Seismic applications throughout the life of the reservoir," Oilfield Review, vol. 14, no. 2, pp. 48-65, 2002.

[54] J. Mestayer, B. Cox, P. Wills, D. Kiyashchenko, J. Lopez, M. Costello, S. Bourne, G. Ugueto, R. Lupton, G. Solano et al., "Field trials of distributed acoustic sensing for geophysical monitoring," in Seg technical program expanded abstracts 2011. Society of Exploration Geophysicists, 2011, pp. 4253-4257.

[55] M. M. Molenaar, D. Hill, P. Webster, E. Fidan, and B. Birch, "First downhole application of distributed acoustic sensing for hydraulicfracturing monitoring and diagnostics," SPE Drilling \& Completion, vol. 27, no. 01, pp. 32-38, 2012.

[56] B. Hardage, "An examination of tube wave noise in vertical seismic profiling data," Geophysics, vol. 46, no. 6, pp. 892-903, 1981.

[57] K. Harris, D. White, and C. Samson, "Imaging the aquistore reservoir after 36 kilotonnes of co2 injection using distributed acoustic sensing," Geophysics, vol. 82, no. 6, pp. M81-M96, 2017.

[58] J. D. Munn, T. I. Coleman, B. L. Parker, M. J. Mondanos, and A. Chalari, "Novel cable coupling technique for improved shallow distributed acoustic sensor vsps," Journal of Applied Geophysics, vol. 138, pp. 72-79, 2017.

[59] R. Barati and J.-T. Liang, "A review of fracturing fluid systems used for hydraulic fracturing of oil and gas wells," Journal of Applied Polymer Science, vol. 131, no. 16, 2014.

[60] G. K. Ekechukwu and J. Sharma, "Well-scale demonstration of distributed pressure sensing using fiber-optic das and dts," Scientific reports, vol. 11, no. 1, pp. 1-18, 2021.

[61] J. Hull, L. Gosselin, and K. Borzel, "Well integrity monitoring \& analysis using distributed acoustic fiber optic sensors," in IADC/SPE Drilling Conference and Exhibition. OnePetro, 2010. 
[62] J. Tejedor, J. Macias-Guarasa, H. F. Martins, J. Pastor-Graells, P. Corredera, and S. Martin-Lopez, "Machine learning methods for pipeline surveillance systems based on distributed acoustic sensing: A review," Applied Sciences, vol. 7, no. 8, p. 841, 2017.

[63] J. Dakin, "Temperature measuring arrangement," UK patent, GB2140554, 1984

[64] M. A. Soto, T. Nannipieri, A. Signorini, A. Lazzeri, F. Baronti, R. Roncella, G. Bolognini, and F. Di Pasquale, "Raman-based distributed temperature sensor with $1 \mathrm{~m}$ spatial resolution over $26 \mathrm{~km}$ smf using low-repetition-rate cyclic pulse coding," Optics letters, vol. 36, no. 13 , pp. 2557-2559, 2011.

[65] Y. Liu, L. Ma, C. Yang, W. Tong, and Z. He, "Long-range raman distributed temperature sensor with high spatial and temperature resolution using graded-index few-mode fiber," Optics express, vol. 26, no. 16, pp. 20 562-20571, 2018.

[66] M. Höbel, J. Ricka, M. Wüthrich, and T. Binkert, "High-resolution distributed temperature sensing with the multiphoton-timing technique," Applied optics, vol. 34, no. 16, pp. 2955-2967, 1995.

[67] G. P. Agrawal, "Nonlinear fiber optics," in Nonlinear Science at the Dawn of the 21st Century. Springer, 2000, pp. 195-211.

[68] G. A. Brown, "The essentials of fiber-optic distributed temperature analysis." Schlumberger Educational Services, 2005.

[69] J. J. Smolen, "Distributed temperature sensing, a dts primer for oil \& gas production," EP2003, 5, 2003.

[70] G. A. Brown, B. Kennedy, and T. Meling, "Using fibre-optic distributed temperature measurements to provide real-time reservoir surveillance data on wytch farm field horizontal extended-reach wells," in SPE Annual Technical Conference and Exhibition. OnePetro, 2000.

[71] M. Abbaszadeh and M. Kamal, "Pressure-transient testing of waterinjection wells," SPE reservoir engineering, vol. 4, no. 01, pp. 115124, 1989.

[72] G. A. Brown and A. Hartog, "Optical fiber sensors in upstream oil \& gas," Journal of petroleum technology, vol. 54, no. 11, pp. 63-65, 2002.

[73] T. Nowak, "The estimation of water injection profiles from temperature surveys," Journal of Petroleum Technology, vol. 5, no. 08, pp. 203-212, 1953.

[74] R. M. Butler, "Steam-assisted gravity drainage: concept, development, performance and future," Journal of Canadian Petroleum Technology, vol. 33, no. 02, pp. 44-50, 1994.

[75] M. A. Beshry, P. Krawchuk, G. A. Brown, and B. Brough, "Predicting the flow distribution on total e\&p canada's joslyn project horizontal sagd producing wells using permanently installed fiber-optic monitoring," in SPE Annual Technical Conference and Exhibition. OnePetro, 2006.

[76] C. Baldwin, "Applications of fiber optic sensors for heavy oil production," in Fiber Optic Sensors and Applications XIV, vol. 10208 International Society for Optics and Photonics, 2017, p. 102080N.

[77] M. A. Adegboye, W.-K. Fung, and A. Karnik, "Recent advances in pipeline monitoring and oil leakage detection technologies: Principles and approaches," Sensors, vol. 19, no. 11, p. 2548, 2019.

[78] S. P. Siebenaler and G. R. Walter, "Detection of small leaks in liquid pipelines utilizing distributed temperature sensing," in International Pipeline Conference, vol. 45127. American Society of Mechanical Engineers, 2012, pp. 733-744.

[79] T. M. Daley, B. M. Freifeld, J. Ajo-Franklin, S. Dou, R. Pevzner, V. Shulakova, S. Kashikar, D. E. Miller, J. Goetz, J. Henninges et al., "Field testing of fiber-optic distributed acoustic sensing (das) for subsurface seismic monitoring," The Leading Edge, vol. 32, no. 6, pp. 699-706, 2013.

[80] Y. Mao, I. Ashry, F. Hveding, A. Y. Bukhamsin, Y. Hong, T. K. Ng, and B. S. Ooi, "Simultaneous distributed acoustic and temperature sensing using a multimode fiber," IEEE Journal of Selected Topics in Quantum Electronics, vol. 26, no. 4, pp. 1-7, 2020.

[81] F. Hveding, I. Ashry, M. Yuan, B. S. Ooi, and M. Arsalan, "Simultaneous distributed temperature and vibration sensing using multimode optical fiber," 2020, uS Patent 10,880,007.

[82] Z. Haas and M. A. Santoro, "A mode-filtering scheme for improvement of the bandwidth-distance product in multimode fiber systems," Journal of lightwave technology, vol. 11, no. 7, pp. 1125-1131, 1993.

[83] D. H. Sim, Y. Takushima, and Y. C. Chung, "High-speed multimode fiber transmission by using mode-field matched center-launching technique," Journal of Lightwave Technology, vol. 27, no. 8, pp. 10181026, 2009.

[84] Z. Wang, H. Wu, X. Hu, N. Zhao, Q. Mo, and G. Li, "Rayleigh scattering in few-mode optical fibers," Scientific reports, vol. 6, no. 35844 , pp. 1-8, 2016.
[85] C. A. Galindez-Jamioy and J. M. Lopez-Higuera, "Brillouin distributed fiber sensors: an overview and applications," Journal of Sensors, vol. 2012, 2012.

[86] K. Kishida, Y. Yamauchi, and A. Guzik, "Study of optical fibers strain-temperature sensitivities using hybrid brillouin-rayleigh system,' Photonic sensors, vol. 4, no. 1, pp. 1-11, 2014.

[87] M. Alahbabi, Y. Cho, and T. Newson, "Simultaneous temperature and strain measurement with combined spontaneous raman and brillouin scattering," Optics letters, vol. 30, no. 11, pp. 1276-1278, 2005

[88] S. M. Maughan, H. H. Kee, and T. P. Newson, "Simultaneous distributed fibre temperature and strain sensor using microwave coherent detection of spontaneous brillouin backscatter," Measurement Science and Technology, vol. 12, no. 7, p. 834, 2001

[89] X. Bao, Q. Yu, and L. Chen, "Simultaneous strain and temperature measurements with polarization-maintaining fibers and their error analysis by use of a distributed brillouin loss system," Optics letters, vol. 29, no. 12 , pp. 1342-1344, 2004.

[90] W. Zou, Z. He, and K. Hotate, "Complete discrimination of strain and temperature using brillouin frequency shift and birefringence in a polarization-maintaining fiber," Optics express, vol. 17, no. 3, pp. 1248-1255, 2009.

[91] Z. Zhao, Y. Dang, M. Tang, B. Li, L. Gan, S. Fu, H. Wei, W. Tong, P. Shum, and D. Liu, "Spatial-division multiplexed brillouin distributed sensing based on a heterogeneous multicore fiber," Optics letters, vol. 42, no. 1, pp. 171-174, 2017.

[92] L. Zou, X. Bao, S. Afshar, and L. Chen, "Dependence of the brillouin frequency shift on strain and temperature in a photonic crystal fiber," Optics letters, vol. 29, no. 13, pp. 1485-1487, 2004.

[93] X. Liu and X. Bao, "Brillouin spectrum in leaf and simultaneous temperature and strain measurement," Journal of Lightwave Technology, vol. 30, no. 8, pp. 1053-1059, 2011.

[94] Z. Li, L. Yan, X. Zhang, and W. Pan, "Temperature and strain discrimination in botda fiber sensor by utilizing dispersion compensating fiber," IEEE Sensors Journal, vol. 18, no. 17, pp. 7100-7105, 2018.

[95] A. Li, Y. Wang, J. Fang, M.-J. Li, B. Y. Kim, and W. Shieh, "Fewmode fiber multi-parameter sensor with distributed temperature and strain discrimination," Optics letters, vol. 40, no. 7, pp. 1488-1491, 2015.

[96] F. Ravet, F. Briffod, S. Chin, E. Rochat, and J.-G. Martinez, "Pipeline geohazard risk monitoring with optical fiber distributed sensors: Experience with andean and arctic routes," in International Pipeline Conference, vol. 51883. American Society of Mechanical Engineers, 2018, p. V003T04A031

[97] F. Ravet, C. Borda, E. Rochat, and M. Niklès, "Geohazard prevention and pipeline deformation monitoring using distributed optical fiber sensing," in ASME International Pipeline Geotechnical Conference, vol. 55775. American Society of Mechanical Engineers, 2013, p. V001T04A001.

[98] M. Nikles, B. H. Vogel, F. Briffod, S. Grosswig, F. Sauser, S. Luebbecke, A. Bals, and T. Pfeiffer, "Leakage detection using fiber optics distributed temperature monitoring," in Smart Structures and Materials 2004: Smart Sensor Technology and Measurement Systems, vol. 5384. International Society for Optics and Photonics, 2004, pp. $18-25$.

[99] M. Niklès, F. Briffod, R. Burke, and G. Lyons, "Greatly extended distance pipeline monitoring using fibre optics," in International Conference on Offshore Mechanics and Arctic Engineering, vol. 41979, 2005, pp. 539-546.

[100] D. Inaudi and B. Glisic, "Application of distributed fiber optic sensory for shm," Proceedings of the ISHMII-2, vol. 1, pp. 163-169, 2005.

[101] M. Nikles, "Long-distance fiber optic sensing solutions for pipeline leakage, intrusion, and ground movement detection," in Fiber optic sensors and applications VI, vol. 7316. International Society for Optics and Photonics, 2009, p. 731602.

[102] S. Z. Yan and L. S. Chyan, "Performance enhancement of botdr fiber optic sensor for oil and gas pipeline monitoring," Optical Fiber Technology, vol. 16, no. 2, pp. 100-109, 2010.

[103] B. Glisic and Y. Yao, "Fiber optic method for health assessment of pipelines subjected to earthquake-induced ground movement," Structural Health Monitoring, vol. 11, no. 6, pp. 696-711, 2012.

[104] P. Rajeev, J. Kodikara, W. K. Chiu, and T. Kuen, "Distributed optical fibre sensors and their applications in pipeline monitoring," in Key Engineering Materials, vol. 558. Trans Tech Publ, 2013, pp. 424434.

[105] A. Barrias, J. R. Casas, and S. Villalba, "A review of distributed optical fiber sensors for civil engineering applications," Sensors, vol. 16, no. 5, p. $748,2016$. 
[106] T. Sasaki, J. Park, K. Soga, T. Momoki, K. Kawaguchi, H. Muramatsu, Y. Imasato, A. Balagopal, J. Fontenot, and T. Hall, "Distributed fibre optic strain sensing of an axially deformed well model in the laboratory," Journal of Natural Gas Science and Engineering, vol. 72, p. 103028, 2019.

[107] T. Horiguchi and M. Tateda, "Botda-nondestructive measurement of single-mode optical fiber attenuation characteristics using brillouin interaction: theory," Journal of lightwave technology, vol. 7, no. 8, pp. 1170-1176, 1989.

[108] D. Culverhouse, F. Farahi, C. Pannell, and D. Jackson, "Potential of stimulated brillouin scattering as sensing mechanism for distributed temperature sensors," Electronics Letters, vol. 25, no. 14, pp. 913-915, 1989.

[109] T. Horiguchi, T. Kurashima, and M. Tateda, "Tensile strain dependence of brillouin frequency shift in silica optical fibers," IEEE Photonics Technology Letters, vol. 1, no. 5, pp. 107-108, 1989.

[110] T. Kurashima, T. Horiguchi, H. Izumita, S.-i. Furukawa, and Y. Koyamada, "Brillouin optical-fiber time domain reflectometry," IEICE transactions on communications, vol. 76, no. 4, pp. 382-390, 1993.

[111] X. Bao, D. J. Webb, and D. A. Jackson, "22-km distributed temperature sensor using brillouin gain in an optical fiber," Optics letters, vol. 18, no. 7, pp. 552-554, 1993.

[112] H. H. Kee, G. P. Lees, and T. P. Newson, "All-fiber system for simultaneous interrogation of distributed strain and temperature sensing by spontaneous brillouin scattering," Optics letters, vol. 25, no. 10, pp. 695-697, 2000.

[113] H. Liang, W. Li, N. Linze, L. Chen, and X. Bao, "High-resolution dpp-botda over $50 \mathrm{~km}$ leaf using return-to-zero coded pulses," Optics letters, vol. 35, no. 10, pp. 1503-1505, 2010.

[114] C. N. Pannell, J. Dhliwayo, and D. J. Webb, "The accuracy of parameter estimation from noisy data, with application to resonance peak estimation in distributed brillouin sensing," Measurement science and technology, vol. 9, no. 1, p. 50, 1998.

[115] Y. Antman, A. Clain, Y. London, and A. Zadok, "Optomechanical sensing of liquids outside standard fibers using forward stimulated brillouin scattering," Optica, vol. 3, no. 5, pp. 510-516, 2016.

[116] N. Hayashi, Y. Mizuno, K. Nakamura, S. Y. Set, and S. Yamashita "Experimental study on depolarized gawbs spectrum for optomechanical sensing of liquids outside standard fibers," Optics express, vol. 25, no. 3, pp. 2239-2244, 2017

[117] N. Hayashi, Y. Mizuno, H. Lee, K. Nakamura, S. Y. Set, and S. Yamashita, "Characterization of cascaded forward brillouin scattering seeded by backward stimulated brillouin scattering in optical fibers," IEICE Electronics Express, pp. 17-20200 139, 2020.

[118] C. Pang, Z. Hua, D. Zhou, H. Zhang, L. Chen, X. Bao, and Y. Dong, "Opto-mechanical time-domain analysis based on coherent forward stimulated brillouin scattering probing," Optica, vol. 7, no. 2, pp. 176184, 2020.

[119] N. Hayashi, Y. Mizuno, K. Nakamura, C. Zhang, L. Jin, S. Y. Set, and S. Yamashita, "Pilot demonstration of correlation-domain distributed temperature sensing using forward brillouin scattering," Japanese Journal of Applied Physics, vol. 59, no. 8, p. 088002, 2020.

[120] R. Shelby, M. Levenson, and P. Bayer, "Guided acoustic-wave brillouin scattering," Physical Review B, vol. 31, no. 8, p. 5244, 1985.

[121] A. J. Poustie, "Bandwidth and mode intensities of guided acousticwave brillouin scattering in optical fibers," JOSA B, vol. 10, no. 4, pp. 691-696, 1993.

[122] R. T. Ramos and W. D. Hawthorne, "Survivability of optical fiber for harsh environments," in SPE Annual Technical Conference and Exhibition. OnePetro, 2008.

[123] L. Yang, D. S. Bale, D. Yang, M. Raum, O. Bello, R. Failla, D. Lerohl, D. Knowles, A. Kwari, M. Cannon et al., "Enabling real-time asset analytics for a cloud-based fiber-optic data management system," in SPE Annual Technical Conference and Exhibition. OnePetro, 2018.

[124] X. Bao and L. Chen, "Recent progress in brillouin scattering based fiber sensors," Sensors, vol. 11, no. 4, pp. 4152-4187, 2011.

[125] B. Lee, "Review of the present status of optical fiber sensors," Optical fiber technology, vol. 9, no. 2, pp. 57-79, 2003.

[126] Y. Zhang, G. Keiser, C. Marzinsky, A. M. Schilowitz, L. Song, and A. B. Herhold, "Applications of optical fiber sensors in the oil refining and petrochemical industries," in SENSORS, 2011 IEEE. IEEE, 2011, pp. 246-249.

[127] J. Ballato and L. Dong, "Sixth international workshop on specialty optical fibers and their applications (wsof 2019)," 2019.

[128] M. Boering, R. Braal, and L. K. Cheng, "Toward the next fiber optic revolution and decision making in the oil and gas industry." in Offshore Technology Conference. OnePetro, 2013.
[129] J. Van Der Horst, J. L. Lopez, W. Berlang, and H. Potters, "In-well distributed fiber optic solutions for reservoir surveillance," in Offshore Technology Conference. OnePetro, 2013.

[130] K. Gohari, H. Jutila, A. Kshirsagar, A. Chattopadhyay, C. Mascagnini, A. Gryaznov, P. Kidd, and F. Zarei, "Das/dts/dss/dps/dxs-do we measure what adds value?" in SPE Europec featured at 78th EAGE Conference and Exhibition. OnePetro, 2016.

[131] J. M. Koelman, J. L. Lopez, and J. H. Potters, "Optical fibers: the neurons for future intelligent wells," in SPE Intelligent Energy International. OnePetro, 2012.

[132] K. Balaji, M. Rabiei, V. Suicmez, C. H. Canbaz, Z. Agharzeyva, S. Tek, U. Bulut, and C. Temizel, "Status of data-driven methods and their applications in oil and gas industry," in SPE Europec featured at 80th EAGE Conference and Exhibition. OnePetro, 2018.

[133] O. Bello, D. Yang, S. Lazarus, X. S. Wang, and T. Denney, "Next generation downhole big data platform for dynamic data-driven well and reservoir management," in SPE Reservoir Characterisation and Simulation Conference and Exhibition. OnePetro, 2017.

[134] X. Yang, O. Bello, L. Yang, D. Bale, and R. Failla, "Intelligent oilfieldcloud based big data service in upstream oil and gas," in International Petroleum Technology Conference. OnePetro, 2019.

[135] Q. Song, H. Peng, S. Zhou, P. Zhou, Q. Xiao, and B. Jia, "A novel weak-scattering michelson interferometer based on pbs for longdistance disturbance localization," Journal of Lightwave Technology, vol. 38, no. 6, pp. 1543-1549, 2019.

[136] B. Kizlik, "Fibre optic distributed sensor in mach-zehnder interferometer configuration," in Modern Problems of Radio Engineering, Telecommunications and Computer Science (IEEE Cat. No. 02EX542). IEEE, 2002, pp. 128-130.

[137] C. Ma, T. Liu, K. Liu, J. Jiang, Z. Ding, L. Pan, and M. Tian, "Long-range distributed fiber vibration sensor using an asymmetric dual mach-zehnder interferometers," Journal of Lightwave Technology, vol. 34, no. 9, pp. 2235-2239, 2016.

[138] Q. Sun, D. Liu, J. Wang, and H. Liu, "Distributed fiber-optic vibration sensor using a ring mach-zehnder interferometer," Optics Communications, vol. 281, no. 6, pp. 1538-1544, 2008.

[139] F. Teng, D. Yi, X. Hong, and X. Li, "Distributed fiber optics disturbance sensor using a dual-sagnac interferometer," Optics letters, vol. 44, no. 20, pp. 5101-5103, 2019.

[140] K. Wada, H. Narui, D. Yamamoto, T. Matsuyama, and H. Horinaka, "Balanced polarization maintaining fiber sagnac interferometer vibration sensor," Optics express, vol. 19, no. 22, pp. 21 467-21 474, 2011.

[141] Y. Li, Z. Liu, Y. Liu, L. Ma, Z. Tan, and S. Jian, "Interferometric vibration sensor using phase-generated carrier method," Applied Optics, vol. 52, no. 25, pp. 6359-6363, 2013.

[142] Q. Song, P. Zhou, H. Peng, Y. Hu, Q. Xiao, H. Wu, and B. Jia, "Improved localization algorithm for distributed fiber-optic sensor based on merged michelson-sagnac interferometer," Optics express, vol. 28, no. 5, pp. 7207-7220, 2020.

[143] S. Wang, P. Lu, L. Mao, D. Liu, and S. Jiang, "Cascaded interferometers structure based on dual-pass mach-zehnder interferometer and sagnac interferometer for dual-parameter sensing," Optics express, vol. 23, no. 2, pp. 674-680, 2015.

[144] S.-C. Huang, W.-W. Lin, M.-T. Tsai, and M.-H. Chen, "Fiber optic in-line distributed sensor for detection and localization of the pipeline leaks," Sensors and Actuators A: Physical, vol. 135, no. 2, pp. 570-579, 2007.

[145] W. T. Lin, S. Q. Lou, and S. Liang, "Fiber-optic distributed vibration sensor for pipeline pre-alarm," in Applied Mechanics and Materials, vol. 684. Trans Tech Publ, 2014, pp. 235-239.

[146] Y. Huang, Q. Wang, L. Shi, and Q. Yang, "Underwater gas pipeline leakage source localization by distributed fiber-optic sensing based on particle swarm optimization tuning of the support vector machine," Applied optics, vol. 55, no. 2, pp. 242-247, 2016. 